\title{
Catalytic CH Arylation of SEM-Protected Azoles Using Palladium Complexes of NHCs and Phosphines.
}

\author{
B. Barry Touré, Benjamin S. Lane, and Dalibor Sames* \\ Department of Chemistry, Columbia University, 3000 Broadway, New York, New York 10027.
}

\author{
Supporting Information
}

\section{General Procedures}

All reactions were performed under an atmosphere of argon, which was passed through Drierite. Nuclear Magnetic Resonance spectra were recorded at 298 K on Bruker Avance DPX 300 and 400 Fourier Transform NMR. Spectra recorded in $\mathrm{CDCl}_{3}$ solutions were referenced to TMS; spectra recorded in other deuterated solvents were referenced to residual solvent peaks. IR spectra were taken as thin films on $\mathrm{NaCl}$ plates using a Perkin-Elmer 1600 FTIR spectrophotometer. Mass spectra were recorded on a JEOL Lcmate instrument (Ionization mode: APCI+). All HPLC analysis was conducted on a Waters Millennium32 Analytical system with a 996 photodiode array detector; all runs were analyzed at $254 \mathrm{~nm}$, with a Zorbax Rx-C18 Column $(4.6 \mathrm{~mm}$ x $25 \mathrm{~cm})$. All HPLC methods were conducted using 30\% acetonitrile / water (with $0.1 \%$ trifluoroacetic acid) with a linear gradient to $100 \%$ acetonitrile over 20 minutes for 1-alkylindoles and 30 min for 1-SEMindoles; this was followed by a 5 minute wash of $100 \%$ acetonitrile and a 5 minute re-equilibration time; analyte concentrations were determined versus benzoic acid as an internal standard. Flash chromatography was carried out on SILICYCLE silica gel (230-400 mesh). Unless otherwise mentioned, all reaction vessels were flame-dried, and cooled to room temperature under an atmosphere of argon.

\section{Materials:}

All materials were used as received unless otherwise noted. 1-Methylindole, purchased from Aldrich, was purified via fractional distillation onto $4 \AA$ molecular sieves; the purified material was stored under argon in the dark prior to use. Electron rich SEM-indoles slowly decompose upon standing, and were passed through a short silica plug, and dried under high vacuum prior to use. Their arylation products are stable and can be kept at room temperature for a long period of time.

3. Synthesis of the palladium complexes (The stereochemistry of these compounds still remain to be established)

\subsection{Complex 1a}

2 (Scheme 1) was prepared using a known protocol, ${ }^{1}$ and alkylated using 2-iodopropane according to the following procedure:

$2(0.4 \mathrm{~g}, 2.5 \mathrm{mmol})$ was dissolved in acetonitrile $(10.0 \mathrm{~mL})$ and treated with 2-iodopropane $(1.0 \mathrm{~mL}$, $10.0 \mathrm{mmol}$ ). The reaction mixture was refluxed for $40 \mathrm{~h}$, then cooled to room temperature, and the solvent was removed to afford an oily residue, which turned into a white solid upon addition of acetone. 3-pyrrolidinecarbamoyl-1-isopropylimidazolium iodide (3) was collected by filtration, washed extensively with ether, and dried under vacuum $(0.71 \mathrm{~g}, 84 \%)$. 


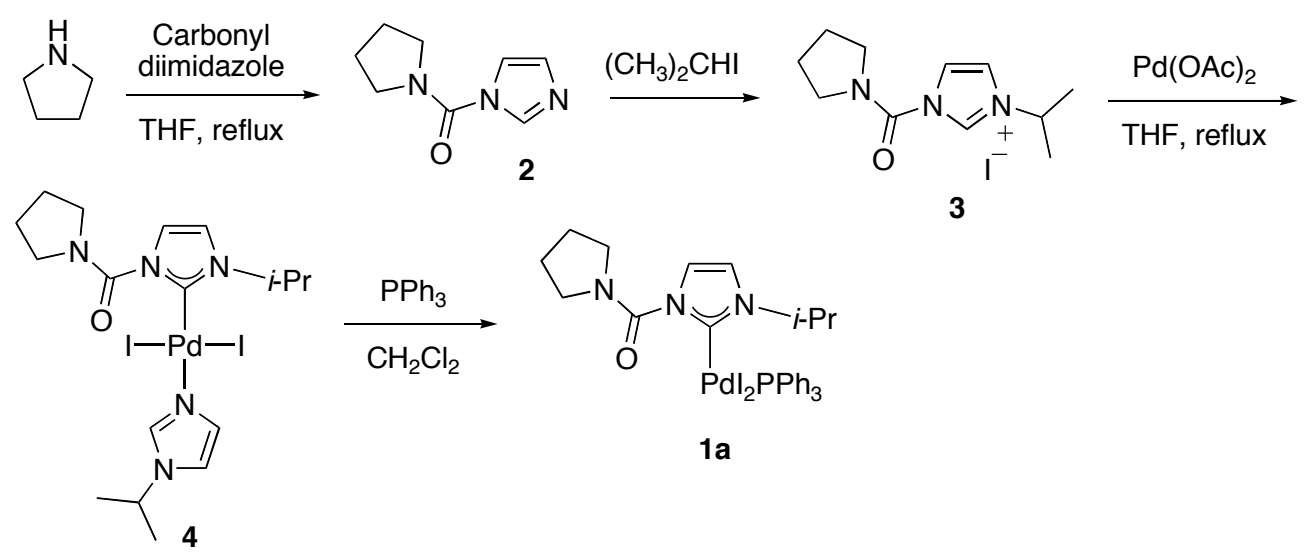

Scheme 1.

1a was obtained from $\mathbf{3}$ in two steps via a known literature procedure. ${ }^{1} \mathbf{4}$ is best purified by silica gel chromatography using ethyl acetate/ hexane (6:4) (70\% from 3, orange solid). Palladium complex 1a is obtained as a yellow solid after silica gel chromatography (69\%). The complex can also be recrystalized by slow diffusion of $\mathrm{Et}_{2} \mathrm{O}$ into a saturated solution of $\mathbf{1 a}$ in $\mathrm{CH}_{2} \mathrm{Cl}_{2}(92 \%$ from 3). Finally, simple stirring of the reaction mixture in hot EtOAc followed by cooling on ice, and filtration efficiently removes the displaced imidazole impurity, and yields pure 1a. TLC (70\% EtOAc/Hexane): 0.3; ${ }^{1} \mathrm{H}$ NMR $\left(\mathrm{CDCl}_{3}, 300 \mathrm{MHz}\right): \delta 8.21-6.70$ (bs, $\left.15 \mathrm{H}\right), 7.08(\mathrm{~d}, J=2.1 \mathrm{~Hz}, 1 \mathrm{H})$, $6.70(\mathrm{~d}, J=2.1 \mathrm{~Hz}, 1 \mathrm{H}), 5.09$ (sept, $J=6.8 \mathrm{~Hz}, 1 \mathrm{H}), 4.02-3.73(\mathrm{~m}, 2 \mathrm{H}), 3.52-3.88$ (bs, $1 \mathrm{H}), 3.32-$ 3.04 (bs, $1 \mathrm{H}), 2.20-1.80(\mathrm{bs}, 4 \mathrm{H}), 1.52(\mathrm{~d}, J=6.8 \mathrm{~Hz}, 3 \mathrm{H}), 0.76(\mathrm{~d}, J=6.8 \mathrm{~Hz}, 3 \mathrm{H})$; Similar to what we have observed with other well characterized compounds of this class, ${ }^{1}$ the pyrrolidine and phosphine signals are very broad $(\mathrm{bs}=$ broad signal $) .{ }^{13} \mathrm{C} \mathrm{NMR}\left(\mathrm{CDCl}_{3}, 100 \mathrm{MHz}\right): \delta 166.7,150.2$, 135.7 (bs), 128.3, 128.2, 121.2, 117.5, 54.2, 50.7, 48.3, 26.7, 25.3, 24.3, 21.9; E.A: calcd for $\mathrm{C}_{29} \mathrm{H}_{32} \mathrm{I}_{2} \mathrm{~N}_{3} \mathrm{OPPd}$ : C (42.00), $\mathrm{H}$ (3.90), N (5.07); found: C (41.86), H (3.80), N (5.13).

\subsection{Complex 1b}

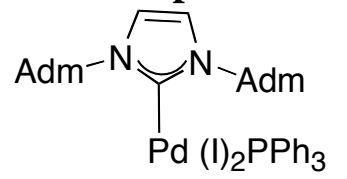

This complex was prepared in $6 \%$ overall yield using the procedure described below for 1d. TLC (30\% $\left.\mathrm{Et}_{2} \mathrm{O} / \mathrm{Hexane}, \mathrm{UV}\right): 0.4 ;{ }^{1} \mathrm{H} \mathrm{NMR}\left(\mathrm{CDCl}_{3}, 400 \mathrm{MHz}\right)$ : ठ 7.77-7.67 (m, 5H), 7.38-7.32 (m, 12H), 2.81-2.75 (m, 12H), 2.29-2.21 (m, $6 \mathrm{H}), 1.80-1.68(\mathrm{~m}, 12 \mathrm{H}) ;{ }^{13} \mathrm{C} \mathrm{NMR}\left(\mathrm{CDCl}_{3}, 100 \mathrm{MHz}\right): \delta 135.2,135.1,133.7$, 133.3, 130.2, 128.1, 128.0, 119.9, 119.8, 61.1, 45.1, 36.5, 30.7; LRMS $\left(\mathrm{FAB}^{+}\right)$: calcd for $\mathrm{C}_{41} \mathrm{H}_{47} \mathrm{I}_{2} \mathrm{~N}_{2} \mathrm{PPd}$ : $958.1\left[\mathrm{M}^{+}\right]$, found: $831.08[\mathrm{M}-\mathrm{I}]^{+}, 704.2$ [M-2I] $]^{+}$; E.A: calcd for $\mathrm{C}_{41} \mathrm{H}_{47} \mathrm{I}_{2} \mathrm{~N}_{2} \mathrm{PPd}$ : C (51.34), $\mathrm{H}$ (4.94), N (2.92); found: C (50.84), H (4.23), N (3.03). Note: the combustion analysis data for carbon and hydrogen are slightly off despite repeated recrystallization.

\subsection{Complex $1 c^{3}$}

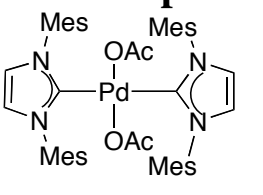

This complex was prepared using a literature procedue. ${ }^{3}{ }^{1} \mathrm{H}$ NMR $\left(\mathrm{CDCl}_{3}, 300\right.$ MHz): $\delta 6.92(\mathrm{~s}, 8 \mathrm{H}), 6.75(\mathrm{~s}, 4 \mathrm{H}), 2.46(\mathrm{~s}, 12 \mathrm{H}), 1.99(\mathrm{~s}, 24 \mathrm{H}), 1.27(\mathrm{~s}, 6 \mathrm{H}) ;{ }^{1} \mathrm{H}$ $\operatorname{NMR}\left(\mathrm{C}_{6} \mathrm{D}_{6}, 300 \mathrm{MHz}\right): \delta 6.90(\mathrm{~s}, 8 \mathrm{H}), 6.04(\mathrm{~s}, 4 \mathrm{H}), 2.33(\mathrm{~s}, 12 \mathrm{H}), 2.17(\mathrm{~s}, 24 \mathrm{H})$, $1.57(\mathrm{~s}, 6 \mathrm{H}) ;{ }^{13} \mathrm{C}$ NMR $\left(\mathrm{CDCl}_{3}, 100 \mathrm{MHz}\right): \delta$ 175.0, 170.1, 137.7, 136.3, 129.1, 122.5, 23.4, 21.9, 19.1; ${ }^{13} \mathrm{C}$ NMR: $\left(\mathrm{C}_{6} \mathrm{D}_{6}, 100 \mathrm{MHz}\right) \delta 174.4,171.2,137.3,136.8,136.4,129.3$, 122.1, 23.3, 21.3, 19.1; LRMS (FAB $^{+}$): found: 773.0 [(IMes) ${ }_{2}$ PdOAc $^{+}, 410.0$ [(IMes)Pd] ${ }^{+}$.

3.4. Complex 1d: (prepared using a modified literature procedure). ${ }^{2}$ 


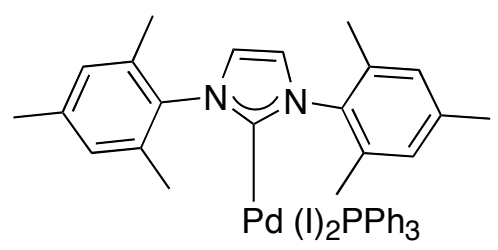

A suspension of 1,3-bis(2,4,6-trimethylphenyl)imidazolium chloride $(0.25 \mathrm{~g}, 0.73 \mathrm{mmol})$, potassium tert-butoxide $95 \%(0.09 \mathrm{~g}, 0.80$ mmol), sodium iodide $(0.44 \mathrm{~g}, 2.90 \mathrm{mmol})$, and palladium acetate $(0.16 \mathrm{~g}, 0.73 \mathrm{mmol})$ in THF $(30.0 \mathrm{~mL})$ was stirred at room temperature for $18 \mathrm{~h}$ in the absence of any light. The solvent was evaporated at room temperature under reduced pressure, and the residue was stirred in ethyl acetate:hexane (6:4) and filtered through a short silica plug. The silica gel was washed with ether, and the solvent was evaporated to afford a red solid, which was further purified by flash chromatography using a mixture of ether and hexane (4:6). This product was dissolved in $\mathrm{CH}_{2} \mathrm{Cl}_{2}$ $(5.0 \mathrm{~mL})$, and treated with triphenylphosphine $(42 \mathrm{mg}, 0.16 \mathrm{mmol})$. The resulting mixture was stirred at room temperature for $2 \mathrm{~h}$, followed by silica gel chromatography using $30 \%$ ether/hexane to afford the product as an orange solid (37 mg, 6\%). TLC (30\% Et 2 O/Hexane, UV): $0.5 ;{ }^{1} \mathrm{H}$ NMR $\left(\mathrm{CDCl}_{3}, 400 \mathrm{MHz}\right): \delta 7.38-7.18(\mathrm{~m}, 15 \mathrm{H}), 7.07(\mathrm{~d}, J=1.4 \mathrm{~Hz}, 2 \mathrm{H}), 7.02(\mathrm{~s}, 4 \mathrm{H}), 2.43(\mathrm{~s}, 6 \mathrm{H}), 2.40$ (s, 12H); ${ }^{13} \mathrm{C} \mathrm{NMR}\left(\mathrm{CDCl}_{3}, 100 \mathrm{MHz}\right): \delta 166.2,164.3,138.9,136.6,136.4,135.8,135.7,134.7$, $134.2,129.9,129.6,127.5,127.4,124.7,124.65,22.7,21.9$; LRMS $\left(\mathrm{FAB}^{+}\right)$: calcd for $\mathrm{C}_{39} \mathrm{H}_{39} \mathrm{I}_{2} \mathrm{~N}_{2} \mathrm{PPd}$ : $926.0\left[\mathrm{M}^{+}\right]$, found: $799.0[\mathrm{M}-\mathrm{I}]^{+}, 672.1[\mathrm{M}-2 \mathrm{I}]^{+}$; E.A: calcd for $\mathrm{C}_{39} \mathrm{H}_{39} \mathrm{I}_{2} \mathrm{~N}_{2} \mathrm{PPd}: \mathrm{C}$ (50.53), H (4.24), N (3.02); found: C (50.73), H (4.44), N (3.01).

\section{Direct comparison of the catalysts (Figure 2).}

In an $8 \mathrm{~mL}$ vial, sealed with an open top closure, $\mathrm{CsOAc}(0.1 \mathrm{~g}, 0.5 \mathrm{mmol})$ was dried under vacuum at $120^{\circ} \mathrm{C}$ over night, and cooled to RT. SEM-indole $(1.6 \mathrm{~g}, 6.5 \mathrm{mmol}), \mathrm{PhI}(0.9 \mathrm{~mL}, 7.8 \mathrm{mmol})$, and dimethyl acetamide (DMA) $(2.6 \mathrm{~mL})$ were mixed in a flame-dried vial. The vials containing dry CsOAc were charged with $0.2 \mathrm{~mL}$ of this solution. A solution of the appropriate catalyst in DMA $(0.1 \mathrm{~mL})$ was added to each vial, and the resulting reaction mixtures were heated to $125^{\circ} \mathrm{C}$. At the time point indicated on Fig. 2, the vials were cooled to $0{ }^{\circ} \mathrm{C}$ then diluted with methanol $(20 \mathrm{~mL})$. This solution was then analyzed by HPLC using benzoic acid as internal standard.

\section{Typical procedure for the protection of the azoles}

2-cyano pyrrole $(0.5 \mathrm{~g}, 5.4 \mathrm{mmol})$ was dissolved in DMF $(10.0 \mathrm{~mL})$ in a flame dried flask under argon, and cooled to $0{ }^{\circ} \mathrm{C}$. $\mathrm{NaH}(240.0 \mathrm{mg}, 6.0 \mathrm{mmol}, 60 \%$ dispersion in mineral oil) was added over $5 \mathrm{~min}$. After $45 \mathrm{~min}$, SEMCl $(5.4 \mathrm{mmol})$ was added slowly, and the reaction was slowly warmed up to room temperature and stirred for $5 \mathrm{~h}$. The reaction was then quenched by slow addition of water, and diluted with ether $(50 \mathrm{~mL})$, water $(25 \mathrm{~mL})$. The two phases were separated and, the aqueous phase was extracted with ether $(2 \times 25 \mathrm{~mL})$. The combined organic phase was washed with water ( 3 X $25 \mathrm{~mL})$, brine $(30 \mathrm{~mL})$, and dried over magnesium sulfate. The solvent was evaporated under reduced pressure and the residue was purified by silica gel chromatography using ethyl acetate/hexane (5:1) to afford the product as a colorless oil in $91 \%$.

\section{Arylation of SEM-indole using Pd(OAc) ${ }_{2}$}

Cesium acetate $(0.1 \mathrm{~g}, 0.5 \mathrm{mmol})$ was dried as described above. To each vial was added SEM-indole (62 mg, $0.25 \mathrm{mmol})$, $\mathrm{PhI}(56.0 \mu \mathrm{L}, 0.5 \mathrm{mmol}) . \mathrm{Pd}(\mathrm{OAc})_{2}(4.8 \mathrm{mg})$, and $\mathrm{PPh}_{3}(22.0 \mathrm{mg})$ were dissolved in DMA $(0.75 \mathrm{~mL})$, and the appropriate amount was added to each vial. The final volume of DMA was adjusted to $0.5 \mathrm{~mL}$ in each vial. The reaction mixtures were heated to $125^{\circ} \mathrm{C}$ for $20 \mathrm{~h}$. 
The yields were established by ${ }^{1} \mathrm{H}$ NMR analysis of the crude reaction mixture using $t$-BuOH as internal standard in the case of indole, and GC for biphenyl using dodecane as internal standard.

Table 1

\begin{tabular}{|c|c|c|}
\hline Cat $(\mathbf{m o l} \%)$ & \% conversion (indole) & ${\text { Yield } \mathbf{( P h - P h ) ~}(\boldsymbol{\%})^{\mathbf{a}}}^{\mathbf{a}}$ \\
\hline 0.5 & 55 & 12 \\
\hline 1.0 & 63 & 18 \\
\hline 2.0 & 69 & 27 \\
\hline 4.0 & 71 & 56 \\
\hline
\end{tabular}

${ }^{\text {a }}$ Yield based on $\mathrm{PhI}$.

\section{Typical Arylation procedure}

In an $8 \mathrm{~mL}$ vial, sealed with an open top closure, CsOAc $(0.2 \mathrm{~g}, 1.0 \mathrm{mmol})$ was dried under vacuum at $120{ }^{\circ} \mathrm{C}$ for $2 \mathrm{~h}$. The vials were then cooled to room temperature under argon, and charged with the appropriate amount of the catalyst. The substrate $(0.5 \mathrm{mmol})$, DMA (volume indicated for each substrate), $\mathrm{PhI}$ were sequentially added, and the resulting reaction mixture was heated to $125^{\circ} \mathrm{C}$ for $24 \mathrm{~h}$, and then cooled to RT. The reaction mixture was diluted with ether ( 25 $\mathrm{mL})$, washed with water $(3 \times 10 \mathrm{~mL})$, brine $(10 \mathrm{~mL})$, and dried over $\mathrm{MgSO}_{4}$. The solvent was evaporated and the residue was purified by silica gel chromatography to afford the desired product.

Note: In all cases Ar-Ar byproducts are observed.

\section{2-Phenyl-1-[2-(trimethylsilyl)-ethoxymethyl]-indole}<smiles>N#[SH]n1c(-c2ccccc2)cc2ccccc21</smiles>

See general procedure: DMA $(0.6 \mathrm{~mL}), \mathrm{PhI}(1.5$ equiv $)$, substrate $(2.0 \mathrm{mmol})$, CsOAc $(2.0 \mathrm{mmol})$. TLC (9\% Et $\mathrm{O} /$ Hexane, UV): 0.51; ${ }^{1} \mathrm{H} \mathrm{NMR}\left(\mathrm{CDCl}_{3}, 300\right.$ $\mathrm{MHz}): \delta 7.66(\mathrm{~m}, 3 \mathrm{H}), 7.56-7.31(\mathrm{~m}, 4 \mathrm{H}), 7.23(\mathrm{dd}, J=6.6,6.7 \mathrm{~Hz}, 1 \mathrm{H}), 7.20$ (dd, $J=6.7,6.9 \mathrm{~Hz}, 1 \mathrm{H}), 6.64(\mathrm{~s}, 1 \mathrm{H}), 5.51(\mathrm{~s}, 2 \mathrm{H}), 3.54(\mathrm{t}, J=8.0 \mathrm{~Hz}, 2 \mathrm{H}), 0.94(\mathrm{t}, J=$ $8.0 \mathrm{~Hz}, 2 \mathrm{H}),-0.1(\mathrm{~s}, 9 \mathrm{H}) ;{ }^{13} \mathrm{C} \mathrm{NMR}\left(\mathrm{CDCl}_{3}, 100 \mathrm{MHz}\right): \delta 142.0,138.6,132.9,129.9,128.9,128.7$, 128.4, 122.6, 121.0, 120.9, 110.7, 103.6, 73.4, 66.3, 18.6, 0.0; IR ( $\mathrm{CDCl}_{3}$, film): $v_{\max } 3082,3060$, 3025, 2924, 2849, 1942, 1871, 1802, 1745, 1601, 1493, 1451, 1372, $1028 \mathrm{~cm}^{-1}$; LRMS (APCI): calcd for $\mathrm{C}_{20} \mathrm{H}_{25} \mathrm{NOSi}$ : 323.17 , found: $324.11[\mathrm{M}+\mathrm{H}]^{+}$.

\section{2-Phenyl-1-[2-(trimethylsilyl)-ethoxymethyl]-indole-5-carbonitrile}<smiles>N#Cc1ccc2c(c1)cc(-c1ccccc1)n2S</smiles>

See general procedure: DMA $(0.3 \mathrm{~mL}), \mathrm{PhI}$ (1.4 equiv), substrate $(0.5 \mathrm{mmol})$. TLC (10\% EtOAc/Hexane, UV): 0.30; ${ }^{1} \mathrm{H} \mathrm{NMR}\left(\mathrm{CDCl}_{3}, 300 \mathrm{MHz}\right): \delta 8.0(\mathrm{~d}$, $J=1.1 \mathrm{~Hz}, 1 \mathrm{H}), 7.69-7.51(\mathrm{~m}, 7 \mathrm{H}), 6.68(\mathrm{~s}, 1 \mathrm{H}), 5.51(\mathrm{~s}, 2 \mathrm{H}), 3.53(\mathrm{t}, J=8.1$, $2 \mathrm{H}), 0.90(\mathrm{t}, J=8.1 \mathrm{~Hz}, 2 \mathrm{H}),-0.2(\mathrm{~s}, 9 \mathrm{H}) ;{ }^{13} \mathrm{C} \mathrm{NMR}\left(\mathrm{CDCl}_{3}, 100 \mathrm{MHz}\right): \delta$ $144.3,140.0,131.5,129.9,129.3,129.1,126.2,125.4,121.0,111.8,111.7,104.2,103.8,73.5,66.7$, 18.5, 0.0; LRMS (APCI): calcd for $\mathrm{C}_{21} \mathrm{H}_{24} \mathrm{~N}_{2} \mathrm{OSi}$ : 348.13, found: $349.10[\mathrm{M}+\mathrm{H}]^{+}$. The product is contaminated by a small amount of inseparable impurity.

5-Nitro-2-phenyl-1-[2-(trimethylsilyl)-ethoxymethyl]-indole 


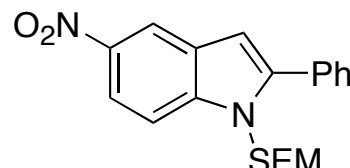

See general procedure: DMA (0.6 mL), PhI (1.4 equiv), substrate $(1 \mathrm{mmol})$. TLC (20\% EtOAc/Hexane, UV): $0.41 ;{ }^{1} \mathrm{H}$ NMR $\left(\mathrm{CDCl}_{3}, 300 \mathrm{MHz}\right): \delta 8.60$ $(\mathrm{d}, J=2.2 \mathrm{~Hz}, 1 \mathrm{H}), 8.19(\mathrm{dd}, J=2.2,9.0 \mathrm{~Hz}, 1 \mathrm{H}), 7.67-7.53(\mathrm{~m}, 6 \mathrm{H}), 6.77(\mathrm{~s}$, $1 \mathrm{H}), 5.50(\mathrm{~s}, 2 \mathrm{H}), 3.5(\mathrm{t}, J=8.1 \mathrm{~Hz}, 2 \mathrm{H}), 0.93(\mathrm{t}, J=8.1 \mathrm{~Hz}, 2 \mathrm{H}),-0.1(\mathrm{~s}$, 9H); $\left.{ }^{13} \mathrm{C} \mathrm{NMR} \mathrm{(} \mathrm{CDCl}_{3}, 100 \mathrm{MHz}\right): \delta 150.0,143.3,142.4,132.5,131.7,129.7,129.0,128.8,128.4$, 120.9, 117.3, 101.2, 71.2, 66.9, 18.7, 0.0; IR ( $\mathrm{CDCl}_{3}$, film): $v_{\max }$ 2952, 2901, 1611, 1514, 1468, 1394, 1336, 1249, 1071 $\mathrm{cm}^{-1}$; LRMS (APCI): calcd for $\mathrm{C}_{20} \mathrm{H}_{24} \mathrm{~N}_{2} \mathrm{O}_{3} \mathrm{Si}: 368.16$, found: $369.10[\mathrm{M}+$ $\mathrm{H}]^{+}$.

\section{3-o-Tolyl-1-[2-(trimethylsilyl)-ethoxymethyl]-indole}

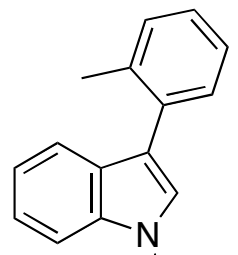

See general procedure: DMA $(0.5 \mathrm{~mL}), 2$-iodotoluene $(2.0$ equiv), substrate $(0.5$ mmol). TLC ( $3 \%$ EtOAc/Hexane, UV): $0.21 ;{ }^{1} \mathrm{H} \mathrm{NMR}\left(\mathrm{CDCl}_{3}, 300 \mathrm{MHz}\right): \delta 7.58$ $(\mathrm{d}, J=7.2 \mathrm{~Hz}, 1 \mathrm{H}), 7.54(\mathrm{~d}, J=7.1 \mathrm{~Hz}, 1 \mathrm{H}), 7.47-7.42(\mathrm{~m}, 1 \mathrm{H}), 7.38-7.28(\mathrm{~m}, 4 \mathrm{H})$, 7.22-7.18 (m, 2H), $5.53(\mathrm{~s}, 2 \mathrm{H}), 3.55(\mathrm{t}, J=8.0 \mathrm{~Hz} .2 \mathrm{H}), 2.35(\mathrm{~s}, 3 \mathrm{H}), 0.93(\mathrm{t}, J=8.0$ $\mathrm{Hz}, 2 \mathrm{H}),-0.3$ (s, 9H); ${ }^{13} \mathrm{C}$ NMR $\left(\mathrm{CDCl}_{3}, 100 \mathrm{MHz}\right): \delta 137.1,136.7,134.4,131.2$, SEM $130.7,128.9,127.2,126.9,126.0,122.6,120.7,120.6,117.5,110.5,76.2,66.4,21.4$, 18.5, -0.1; IR ( $\mathrm{CDCl}_{3}$, film): $v_{\max } 3058,2952,2894,1460,1334,1309,1249,1077$; LRMS (APCI) calcd for $\mathrm{C}_{21} \mathrm{H}_{27} \mathrm{NOSi}$ : 337.19 , found 338.28.

\section{2-o-Tolyl-1-[2-(trimethylsilyl)-ethoxymethyl]-indole}

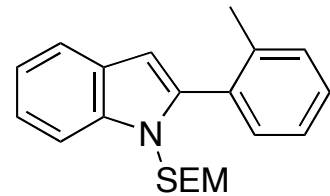

TLC (3 \% EtOAc/Hexane, UV): $0.25 ;{ }^{1} \mathrm{H}$ NMR (CDCl $\left.3,300 \mathrm{MHz}\right): \delta 7.64$ (d, $J=7.6 \mathrm{~Hz}, 1 \mathrm{H}), 7.54(\mathrm{~d}, J=7.2 \mathrm{~Hz}, 1 \mathrm{H}), 7.38-7.18(\mathrm{~m}, 6 \mathrm{H}), 6.45(\mathrm{~s}, 1 \mathrm{H}), 5.28$ (s, $2 \mathrm{H}), 3.24(\mathrm{t}, J=8.1 \mathrm{~Hz}, 2 \mathrm{H}), 2.20(\mathrm{~s}, 3 \mathrm{H}), 0.74(\mathrm{t}, J=8.1 \mathrm{~Hz}, 2 \mathrm{H}),-0.12$ (s. $9 \mathrm{H}) ;{ }^{13} \mathrm{C}$ NMR $\left(\mathrm{CDCl}_{3}, 100 \mathrm{MHz}\right): \delta 140.5,138.5,137.5,132.5,131.9,130.6$, 129.2, 128.9, 125.9, 122.3, 120.9, 120.8, 111.0, 103.7, 73.2, 65.9, 20.6, 18.2, -0.1; IR $\left(\mathrm{CDCL}_{3}\right.$, film): $v_{\max } 3058,2952,2894,1460,1334,1309,1249,1077$; LRMS (APCI) calcd for $\mathrm{C}_{21} \mathrm{H}_{27} \mathrm{NOSi}$ : 337.19, found 338.28. (Note: the reaction mixture is best purified using $4 \% \mathrm{Et}_{2} \mathrm{O}$ in hexane)

\section{2-Phenyl-1-[2-(trimethylsilyl)-ethoxymethyl]-indole-3-carboxylic acid methyl ester}

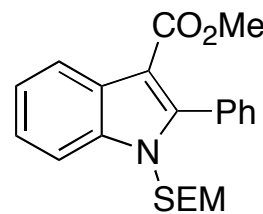

See general procedure: DMA ( $0.25 \mathrm{~mL}), \mathrm{PhI}$ (2.0 equiv), substrate $(0.25 \mathrm{mmol})$. ${ }^{1} \mathrm{H}$ NMR $\left(\mathrm{CDCl}_{3}, 300 \mathrm{MHz}\right): \delta$ 8.26-8.18 $(\mathrm{m}, 1 \mathrm{H}), 7.59-7.40(\mathrm{~m}, 6 \mathrm{H}), 7.38-7.28$ $(\mathrm{m}, 2 \mathrm{H}), 5.32(\mathrm{~s}, 2 \mathrm{H}), 3.76(\mathrm{~s}, 3 \mathrm{H}), 3.32(\mathrm{t}, J=8.1 \mathrm{~Hz}, 2 \mathrm{H}), 0.76(\mathrm{t}, J=8.1 \mathrm{~Hz}$, $2 \mathrm{H}),-0.08$ (s, 9H); ${ }^{13} \mathrm{C}$ NMR $\left(\mathrm{CDCl}_{3}, 100 \mathrm{MHz}\right): \delta 166.0,147.2,136.9,131.4$, 131.1, 129.5, 128.4, 127.1, 123.7. 123.0, 122.4, 111.2, 106.7, 73.3, 66.4, 51.2, 18.1, -.1; LRMS calcd for $\mathrm{C}_{22} \mathrm{H}_{27} \mathrm{NO}_{3}$ Si: 381.1, found $382.12[\mathrm{M}+\mathrm{H}]^{+}$.

3-Methyl-2-phenyl-1H-indole (commercially available)<smiles>Cc1c(-c2ccccc2)[nH]c2ccccc12</smiles>

See general procedure: DMA $(0.3 \mathrm{~mL}), \mathrm{PhI}$ (2.0 equiv), substrate $(0.5 \mathrm{mmol})$. The product was contaminated with inseparable unreacted starting material (15-25\%), thus it was dissolved in THF (5.0 mL), and TBAF (5.0 equiv) was added. After refluxing for $10 \mathrm{~h}$, the solvent was removed, and the residue was purified by silica gel chromatography. TLC (7.5\% EtOAc/Hexane, UV): 0.48; ${ }^{1} \mathrm{H}$ NMR $\left(\mathrm{CDCl}_{3}, 300 \mathrm{MHz}\right): \delta 8.0$ 
(bs, 1H), 7.54-7.46 (m, 3H), $7.40(\mathrm{dd}, J=7.3,7.3 \mathrm{~Hz}, 2 \mathrm{H}), 7.30-7.24(\mathrm{~m}, 2 \mathrm{H}), 7.12(\mathrm{ddd}, J=1.4$, 7.0. 8.1, $1 \mathrm{H}), 7.07(\mathrm{ddd}, J=1.4,7.0 .8 .1,1 \mathrm{H}), 2.41(\mathrm{~s}, 3 \mathrm{H}) ;{ }^{13} \mathrm{C} \mathrm{NMR}\left(\mathrm{CDCl}_{3}, 100 \mathrm{MHz}\right): \delta 136.0$, 134.2, 133.8, 130.4, 129.1, 128.1, 127.6, 122.7, 119.9, 119.3, 111.0, 109.0, 10.2; LRMS (APCI) calcd for $\mathrm{C}_{15} \mathrm{H}_{13} \mathrm{~N}$ : 207.10, found: $208.16[\mathrm{M}+\mathrm{H}]^{+}$.

\section{5-Phenyl-1-[2-(trimethylsilyl)-ethoxymethyl)-pyrrole-2-carbonitrile}

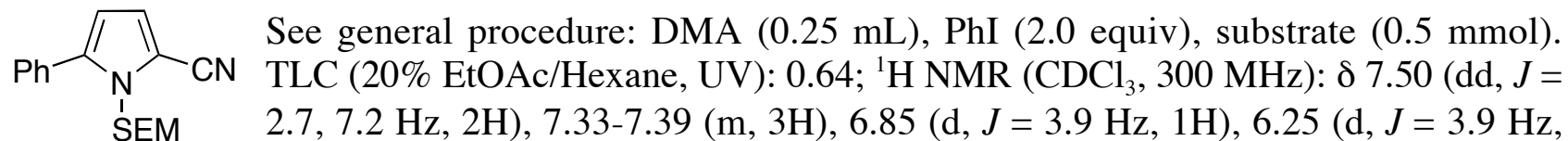
$1 \mathrm{H}), 3.61(\mathrm{t}, J=8.1 \mathrm{~Hz}, 2 \mathrm{H}), 0.90(\mathrm{t}, J=8.1 \mathrm{~Hz}, 2 \mathrm{H}), 0.0(\mathrm{~s}, 9 \mathrm{H}) ;{ }^{13} \mathrm{C} \mathrm{NMR}\left(\mathrm{CDCl}_{3}, 100 \mathrm{MHz}\right): \delta$ 141.2, 131.3, 129.5, 129.1, 129.0, 121.1, 114.5, 110.8, 106.0, 75.0, 67.3, 18.6, 0.0; IR $\left(\mathrm{CDCl}_{3}\right.$, film): $v_{\max } 3064,2953,2896,2215,1604,1460,1398,1311,1249 \mathrm{~cm}^{-1}$; LRMS (APCI): calcd for $\mathrm{C}_{17} \mathrm{H}_{22} \mathrm{~N}_{2} \mathrm{OSi}(298.15)$, found: $299.22[\mathrm{M}+\mathrm{H}]^{+}$. The product is contaminated by a small amount of inseparable impurity (4-6\%).

\section{5-Pyridin-3-yl-1-[2-(trimethylsilyl)-ethoxymethyl]-pyrrole-2-carbonitrile}

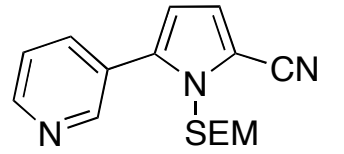

See general procedure: DMA $(0.2 \mathrm{~mL}), 3$-iodopyridine (1.2 equiv), substrate (0.5 mmol). TLC (20\% EtOAc/Hexane, UV): $0.4 ;{ }^{1} \mathrm{H}$ NMR $\left(\mathrm{CDCl}_{3}, 300 \mathrm{MHz}\right)$ $\delta 8.78-8.76(\mathrm{~m}, 1 \mathrm{H}), 8.62(\mathrm{dd}, J=1.5,5.0 \mathrm{~Hz}, 1 \mathrm{H}), 7.95(\mathrm{dm}, J=7.9 \mathrm{~Hz}, 1 \mathrm{H})$, $7.36(\mathrm{dd}, J=5.0,8.0 \mathrm{~Hz}, 1 \mathrm{H}), 6.90(\mathrm{~d}, J=4.0 \mathrm{~Hz}, 1 \mathrm{H}), 6.38(\mathrm{~d}, J=4.0 \mathrm{~Hz}, 1 \mathrm{H}), 5.34(\mathrm{~s}, 2 \mathrm{H}), 3.71$ $(\mathrm{t}, J=8.1 \mathrm{~Hz}, 2 \mathrm{H}), 0.99(\mathrm{t}, J=8.1 \mathrm{~Hz}, 2 \mathrm{H}), 0.0(\mathrm{~s}, 9 \mathrm{H}) ;{ }^{13} \mathrm{C} \mathrm{NMR}\left(\mathrm{CDCl}_{3}, 100 \mathrm{MHz}\right): \delta 150.6$, 150.4, 137.4, 136.5, 127.4, 123.7, 121.0, 114.0, 111.7, 107.2, 75.0, 67.5, 18.6, -0.2; IR $\left(v, \mathrm{~cm}^{-1}\right)$ 2953, 2896, 2218, 1570, 1460, 1428, 1313, 1249, $1085 \mathrm{~cm}^{-1}$; LRMS (APCI): calcd for $\mathrm{C}_{16} \mathrm{H}_{21} \mathrm{~N}_{3} \mathrm{OSi}$ : $299.44\left[\mathrm{M}^{+}\right]$, found: $300.61[\mathrm{M}+\mathrm{H}]^{+}$.

\section{Arylation of [1-(trimethylsilyl)-ethoxymethyl]-pyrrol-3-yl]-ethanone:}

This reaction afforded a mixture of three products, which were assigned structures 5, 6, and $\mathbf{7}$ based on the following NMR data.

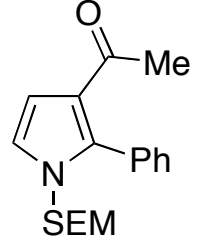

5

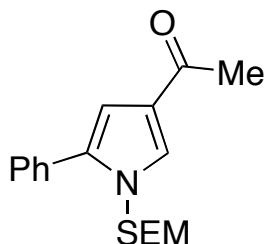

6

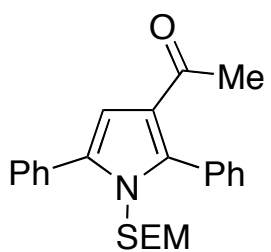

7

5: $J=3.1 \mathrm{~Hz}$ suggested a vicinal relationship between the pyrrole protons.

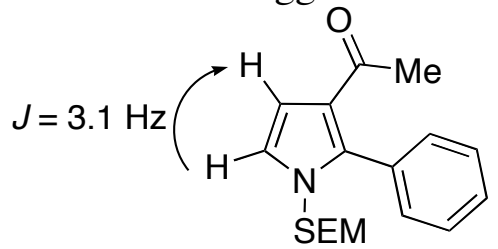

5

6: $J=1.9 \mathrm{~Hz}$ is a typical coupling constant for 1,3 -protons on pyrroles ring. To discriminate between the two possibilities ( $\mathrm{Ph}$ at 4 or 5 positions), nOe studies were conducted. In addition, as 
the $\mathrm{C} 2$ proton in $\mathbf{6}$ was buried under aromatic (phenyl) protons, the product was deprotected and the nOe results were confirmed.

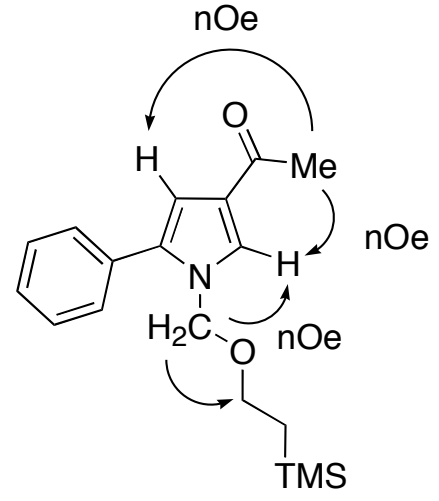

6

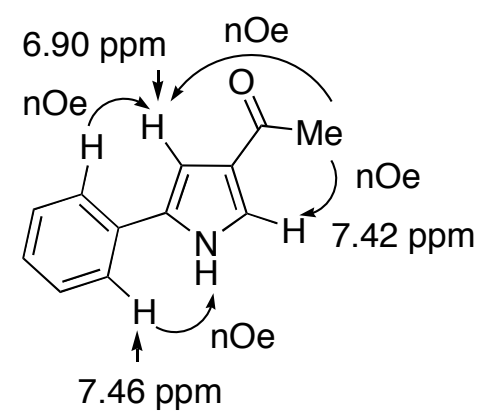

8

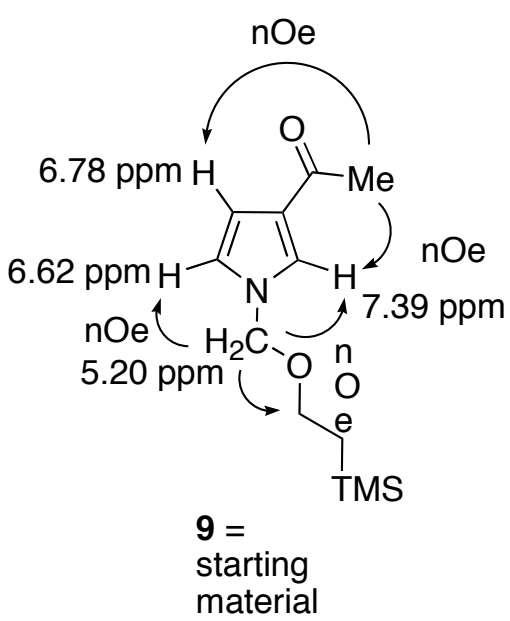

These nOe data are in agreement with the $\mathrm{Ph}$ group being at the 5 position.

Our ${ }^{1} \mathrm{H}-\mathrm{NMR}$ spectrum for $\mathbf{8}$ did not match the literature data. This discrepancy is attributed to a mistake in the latter paper, wherein the ${ }^{1} \mathrm{H}$ NMR spectra for 5-phenyl-3-acetyl pyrrole and 2-phenyl3 -acetyl pyrrole have been inadvertently switched, as confirmed by personal communication with the authors. ${ }^{4}$

7: Based on the above data, it is our assumption that the bis-arylation product results from reaction at the 2 and 5 positions.

The full characterization data are presented below:

\section{1-\{5-Phenyl-1-[2-(trimethylsilyl)-ethoxymethyl]-pyrrol-3-yl\}-ethanone}

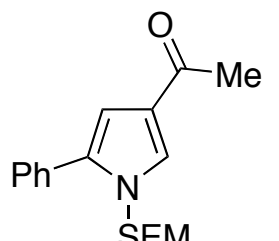

1249, 1177, $1086 \mathrm{~cm}^{-1}$; LRMS (APCI): calcd for $\mathrm{C}_{18} \mathrm{H}_{25} \mathrm{NO}_{2} \mathrm{Si}: 315.17$, found: $316.22[\mathrm{M}+\mathrm{H}]^{+}$.

\section{1-\{2,5-Diphenyl-1-[2-(trimethyl-silyl)-ethoxymethyl]-pyrrol-3-yl\}-ethanone}

See general procedure: DMA $(0.3 \mathrm{~mL})$, PhI (1.4 equiv), substrate $(0.5 \mathrm{mmol})$. TLC (20\%

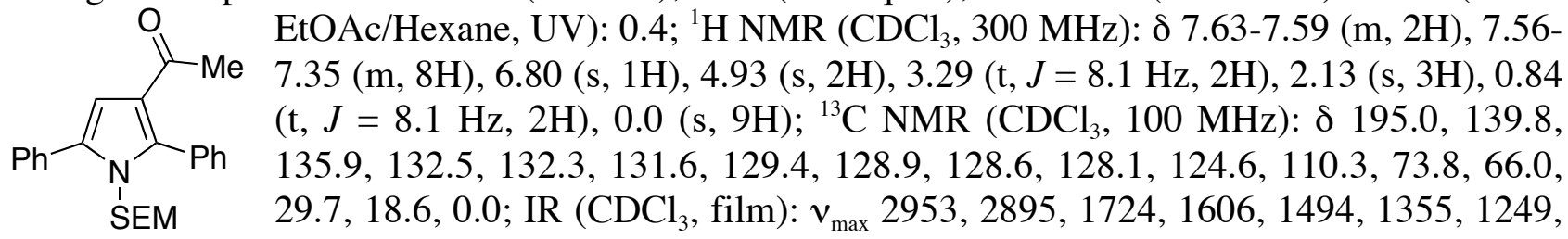
1211, 1087; LRMS (APCI): calcd for $\mathrm{C}_{24} \mathrm{H}_{29} \mathrm{NO}_{2} \mathrm{Si}$ : 391.20, found $392.26[\mathrm{M}+\mathrm{H}]^{+}$. 


\section{1-\{2-Phenyl-1-[2-(trimethylsilyl)-ethoxymethyl]-pyrrol-3-yl\}-ethanone}

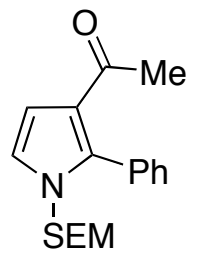

See general procedure: DMA $(0.3 \mathrm{~mL}), \mathrm{PhI}(1.4$ equiv), substrate $(0.5 \mathrm{mmol})$. TLC (20\% EtOAc/Hexane, UV): 0.25; ${ }^{1} \mathrm{H} \mathrm{NMR}\left(\mathrm{CDCl}_{3}, 300 \mathrm{MHz}\right): \delta$ 7.51-7.42 (m, 3H), $7.41-7.37(\mathrm{~m}, 2 \mathrm{H}), 6.84(\mathrm{~d}, J=3.1 \mathrm{~Hz}, 1 \mathrm{H}), 6.75(\mathrm{~d}, J=3.1 \mathrm{~Hz}, 1 \mathrm{H}), 5.0(\mathrm{~s}, 2 \mathrm{H}), 3.39$ (t, $J=8.1 \mathrm{~Hz}, 2 \mathrm{H}), 2.12(\mathrm{~s}, 3 \mathrm{H}), 0.86(\mathrm{t}, J=8.1 \mathrm{~Hz}, 2 \mathrm{H}), 0.0(\mathrm{~s}, 9 \mathrm{H})$; LRMS (APCI) calcd for $\mathrm{C}_{18} \mathrm{H}_{25} \mathrm{NO}_{2} \mathrm{Si}$ : 315.17, found 316.22 (Minor product of the reaction mixture).

\section{2-Phenyl-1-[2-(trimethylsilyl)-ethoxymethyl]-7-azaindole}

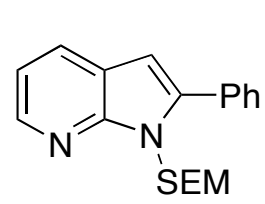

See general procedure: DMA (0.6 mL), PhI (1.4 equiv), substrate (1 mmol). TLC (20\% EtOAc/Hexane, UV): 0.67; ${ }^{1} \mathrm{H}$ NMR $\left(\mathrm{CDCl}_{3}, 300 \mathrm{MHz}\right): \delta 8.39(\mathrm{dd}, J=$ $1.5,4.8 \mathrm{~Hz}, 1 \mathrm{H}), 7.93(\mathrm{dd}, J=1.4,7.7 \mathrm{~Hz}, 1 \mathrm{H}) 7.81(\mathrm{~d}, J=6.8 \mathrm{~Hz}, 2 \mathrm{H}), 7.53-7.48$ $(\mathrm{m}, 3 \mathrm{H}), 7.13(\mathrm{dd}, J=4.7,7.8 \mathrm{~Hz}, 1 \mathrm{H}), 6.61(\mathrm{~s}, 1 \mathrm{H}), 5.52(\mathrm{~s}, 2 \mathrm{H}), 3.74(\mathrm{t}, J=8.2$ $\mathrm{Hz}, 2 \mathrm{H}), 1.01(\mathrm{t}, J=8.2 \mathrm{~Hz}, 2 \mathrm{H}),-0.3(\mathrm{~s}, 9 \mathrm{H}) ;{ }^{13} \mathrm{C} \mathrm{NMR}\left(\mathrm{CDCl}_{3}, 100 \mathrm{MHz}\right): \delta$ $150.0,143.3,142.4,132.5,129.7,129.0,128.8,128.4,120.9,117.3,101.2,71.2,66.9,18.7,0.0$; IR $\left(\mathrm{CDCl}_{3}\right.$, film): $v_{\max } 3061,2951,2894,1572,1477,1426,1249,1073 \mathrm{~cm}^{-1}$. LRMS (APCI): calcd for $\mathrm{C}_{19} \mathrm{H}_{24} \mathrm{~N}_{2} \mathrm{OSi}$ : 324.49 , found: $325.24[\mathrm{M}+\mathrm{H}]^{+}$. The product is contaminated by a small amount of inseparable impurity (See ${ }^{1} \mathrm{H}-\mathrm{NMR}$ spectrum).

\section{5-Phenyl-1-[2-(trimethylsilyl)-ethoxymethyl]-imidazole.}

Ph See general procedure: DMA $(0.5 \mathrm{~mL}), \mathrm{PhI}\left(2.0\right.$ equiv), substrate $(0.5 \mathrm{mmol}) .{ }^{1} \mathrm{H}$ NMR $\left(\mathrm{CDCl}_{3}, 300 \mathrm{MHz}\right): \delta 7.71(\mathrm{~s}, 1 \mathrm{H}), 7.60(\mathrm{~d}, J=6.9 \mathrm{~Hz}, 2 \mathrm{H}) ; 7.48-7.39(\mathrm{~m}, 3 \mathrm{H})$, $\mathrm{N}^{\mathrm{N}}-\mathrm{SEM} 7.19(\mathrm{~s}, 1 \mathrm{H}), 5.42(\mathrm{~s}, 2 \mathrm{H}), 3.57(\mathrm{t}, J=8.2 \mathrm{~Hz}, 2 \mathrm{H}), 0.99(\mathrm{t}, J=8.2 \mathrm{~Hz}, 2 \mathrm{H}), 0.0(\mathrm{~s}$, $9 \mathrm{H}) ;{ }^{13} \mathrm{C} \mathrm{NMR}\left(\mathrm{CDCl}_{3}, 100 \mathrm{MHz}\right): \delta 139.4,133.9,129.8,129.1,129.0,128.8,128.3,74.5,66.8$, 18.4, 0.0; IR $\left(\mathrm{CDCl}_{3}\right.$, film): $v_{\max }$ 3063, 2952, 2894, 1606, 1486, 1484, 1362, 1249, $1075 \mathrm{~cm}^{-1}$; LRMS (APCI): calcd for $\mathrm{C}_{15} \mathrm{H}_{22} \mathrm{~N}_{2} \mathrm{OSi}$ : 274.15, found (LR-APCI) $275.10[\mathrm{M}+\mathrm{H}]^{+}$.

\section{2,5-Diphenyl-1-[2-(trimethylsilyl)-ethoxymethyl]-imidazole.}

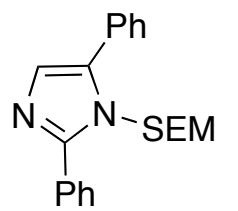

See general procedure: DMA (1.0 mL), PhI (2.0 equiv), substrate $(0.5 \mathrm{mmol})$, $\mathrm{Ag}_{2} \mathrm{CO}_{3}$ (2.0 equiv). TLC (45\% EtOAc/Hexane, UV): $0.55 ;{ }^{1} \mathrm{H}$ NMR $\left(\mathrm{CDCl}_{3}, 300\right.$ $\mathrm{MHz}): \delta 7.80(\mathrm{~d}, J=6.9 \mathrm{~Hz}, 2 \mathrm{H}), 7.59(\mathrm{~d}, J=7.0 \mathrm{~Hz}, 2 \mathrm{H}), 7.49-7.36(\mathrm{~m}, 6 \mathrm{H}), 7.18$ $(\mathrm{s}, 1 \mathrm{H}), 5.42(\mathrm{~s}, 2 \mathrm{H}), 3.45(\mathrm{t}, J=8.1 \mathrm{~Hz}, 2 \mathrm{H}), 0.93(\mathrm{t}, J=8.1 \mathrm{~Hz}, 2 \mathrm{H}), 0.0(\mathrm{~s}, 9 \mathrm{H}) ;{ }^{13} \mathrm{C}$ NMR $\left(\mathrm{CDCl}_{3}, 100 \mathrm{MHz}\right): \delta 150.5,135.7,131.0,130.4,129.3,129.27,129.16,129.1,128.9,128.4$, 127.9, 73.8, 66.0, 18.66, 0.0; IR ( $\mathrm{CDCl}_{3}$, film): $v_{\max }$ 3062, 2952, 2894, 1606, 1486, 1362, 1249 , $1074 \mathrm{~cm}^{-1}$; LRMS calcd for $\mathrm{C}_{21} \mathrm{H}_{26} \mathrm{~N}_{2} \mathrm{OSi}: 350.18$, found: $351.26[\mathrm{M}+\mathrm{H}]^{+}$.

\section{3-Phenyl-imidazo[1,2-a]pyridine}

See general procedure: DMA (0.2 mL), $\mathrm{PhI}$ or $\mathrm{PhBr}(1.2$ equiv), substrate $(0.35 \mathrm{mmol})$. TLC (EtOAc, UV): 0.38; ${ }^{1} \mathrm{H} \mathrm{NMR}\left(\mathrm{CDCl}_{3}, 300 \mathrm{MHz}\right): \delta 8.31$ (d, $\left.J=6.8 \mathrm{~Hz}, 1 \mathrm{H}\right), 7.69(\mathrm{~s}, 1 \mathrm{H}), 7.68(\mathrm{~d}, J$ $=7.2 \mathrm{~Hz}, 1 \mathrm{H}), 7.55-7.39(\mathrm{~m}, 5 \mathrm{H}), 7.17(\mathrm{dd}, J=6.8,7.3 \mathrm{~Hz}, 1 \mathrm{H}), 6.78(\mathrm{dd}, J=6.8,7.0 \mathrm{~Hz}, 1 \mathrm{H}) ;{ }^{13} \mathrm{C}$ $\mathrm{NMR}\left(\mathrm{CDCl}_{3}, 100 \mathrm{MHz}\right): \delta 146.4,132.8,129.6,128.5,128.4,126.1,124.6,123.7,118.6,113.0$; IR 
$\sim \mathrm{N}$

$\left(\mathrm{CDCl}_{3}\right.$, film): $v_{\max } 3056,2982,1636,1604,1500,1482,1354,1298 \mathrm{~cm}^{-1}$; LRMS

(APCI): calcd for $\mathrm{C}_{13} \mathrm{H}_{10} \mathrm{~N}_{2}: 194.08$, found: $195.04[\mathrm{M}+\mathrm{H}]^{+}$.

\section{3-Phenyl-imidazo[1,2-a]pyridine-2-carboxylic acid ethyl ester}<smiles>CCOC(=O)c1nc2ccccn2c1-c1ccccc1</smiles>

See general procedure: DMA (0.6 mL), PhI (1.2 equiv), substrate $(0.5 \mathrm{mmol})$. TLC (EtOAc, UV): 0.49; ${ }^{1} \mathrm{H}$ NMR $\left(\mathrm{CDCl}_{3}, 300 \mathrm{MHz}\right): \delta 7.94(\mathrm{~d}, J=5.2 \mathrm{~Hz}$, $1 \mathrm{H}), 7.73(\mathrm{~d}, J=6.9 \mathrm{~Hz}, 1 \mathrm{H}), 7.29-7.22(\mathrm{~m}, 5 \mathrm{H}), 7.25(\mathrm{dd}, J=5.2,7.0 \mathrm{~Hz}, 1 \mathrm{H})$, $6.80(\mathrm{dd}, J=6.9,7.0 \mathrm{~Hz}, 1 \mathrm{H}), 4.35(\mathrm{q}, J=5.3 \mathrm{~Hz}, 2 \mathrm{H}), 1.30(\mathrm{t}, J=5.3 \mathrm{~Hz} .3 \mathrm{H})$; ${ }^{13} \mathrm{C} \mathrm{NMR}\left(\mathrm{CDCl}_{3}, 100 \mathrm{MHz}\right): \delta 163.8,144.6,133.3,130.4,129.7,129.1,128.4,126.4,124.4,119.4$, 114.0, 61.4, 14.9; IR ( $\mathrm{CDCl}_{3}$, film): $v_{\max }$ 3056, 2982, 1720, 1637, 1508, 1446, 1279, 1234, 1183 $\mathrm{cm}^{-1}$; LRMS (APCI): calcd for $\mathrm{C}_{16} \mathrm{H}_{16} \mathrm{~N}_{2} \mathrm{O}_{2}: 266.11$, found: $267.05[\mathrm{M}+\mathrm{H}]^{+}$.

3-(4-Methoxycarbonylphenyl)-imidazo[1,2-a]pyridine-2-carboxylic acid ethyl ester

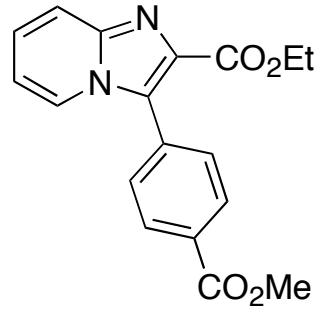

See general procedure: DMA $(0.4 \mathrm{~mL}), 4-\mathrm{BrPhCO}_{2} \mathrm{Me}(1.2$ equiv), substrate (0.5 mmol). TLC (60\% EtOAc/Hexane, UV): $0.15 ;{ }^{1} \mathrm{H} \mathrm{NMR}\left(\mathrm{CDCl}_{3}, 300\right.$ MHz): $\delta 8.23(\mathrm{~d}, J=6.6 \mathrm{~Hz}, 2 \mathrm{H}), 7.92(\mathrm{dd} . J=1.1,7.0 \mathrm{~Hz}, 1 \mathrm{H}), 7.75$ (dd. $J=$ $1.1,7.0 \mathrm{~Hz}, 1 \mathrm{H}), 7.57(\mathrm{~d}, J=6.6 \mathrm{~Hz}, 2 \mathrm{H}), 7.27(\mathrm{~m}, 1 \mathrm{H}), 6.84(\mathrm{dd}, J=6.9,7.0$ $\mathrm{Hz}, 1 \mathrm{H}), 4.38(\mathrm{q}, J=7.1 \mathrm{~Hz}, 2 \mathrm{H}), 3.97(\mathrm{~s}, 3 \mathrm{H}), 1.33(\mathrm{t}, J=7.1 \mathrm{~Hz}, 3 \mathrm{H}) ;{ }^{13} \mathrm{C}$ NMR $\left(\mathrm{CDCl}_{3}, 100 \mathrm{MHz}\right): \delta 166.8,163.0,144.9,133.8,133.0,131.2,131.0$, $130.3,128.4,126.8,124.1,119.5,114.4,61.6,52.9,15.0 ;$ LRMS (APCI): calcd

for $\mathrm{C}_{18} \mathrm{H}_{16} \mathrm{~N}_{2} \mathrm{O}_{4}: 324.11$, found: $325.21[\mathrm{M}+\mathrm{H}]^{+}$.

\section{References}

1. Batey, R. A.; Shen, M.; Lough, A. J. Org. Lett. 2002, 4, 1411-1414.

2. Herrmann, W. A.; Böhm, V. P. W.; Gstöttmayr, C. W. K.; Grosche, M.; Reisinger, C. -P.; Weskamp, T, J. Organomet. Chem. 2001, 617-618, 616-628.

3. Konnick, M. M.; Guzei, I. A.; Stahl, S. S. J. Am. Chem. Soc. 2004, 126, 10212-10213

4. Katritzky, A. R.; Zhu, L.; Lang, H.; Denisko, O.; Wang, Z. Tetrahedron 1995, 51, 13271-13275. 


\section{Compound 1a, Fig.1}
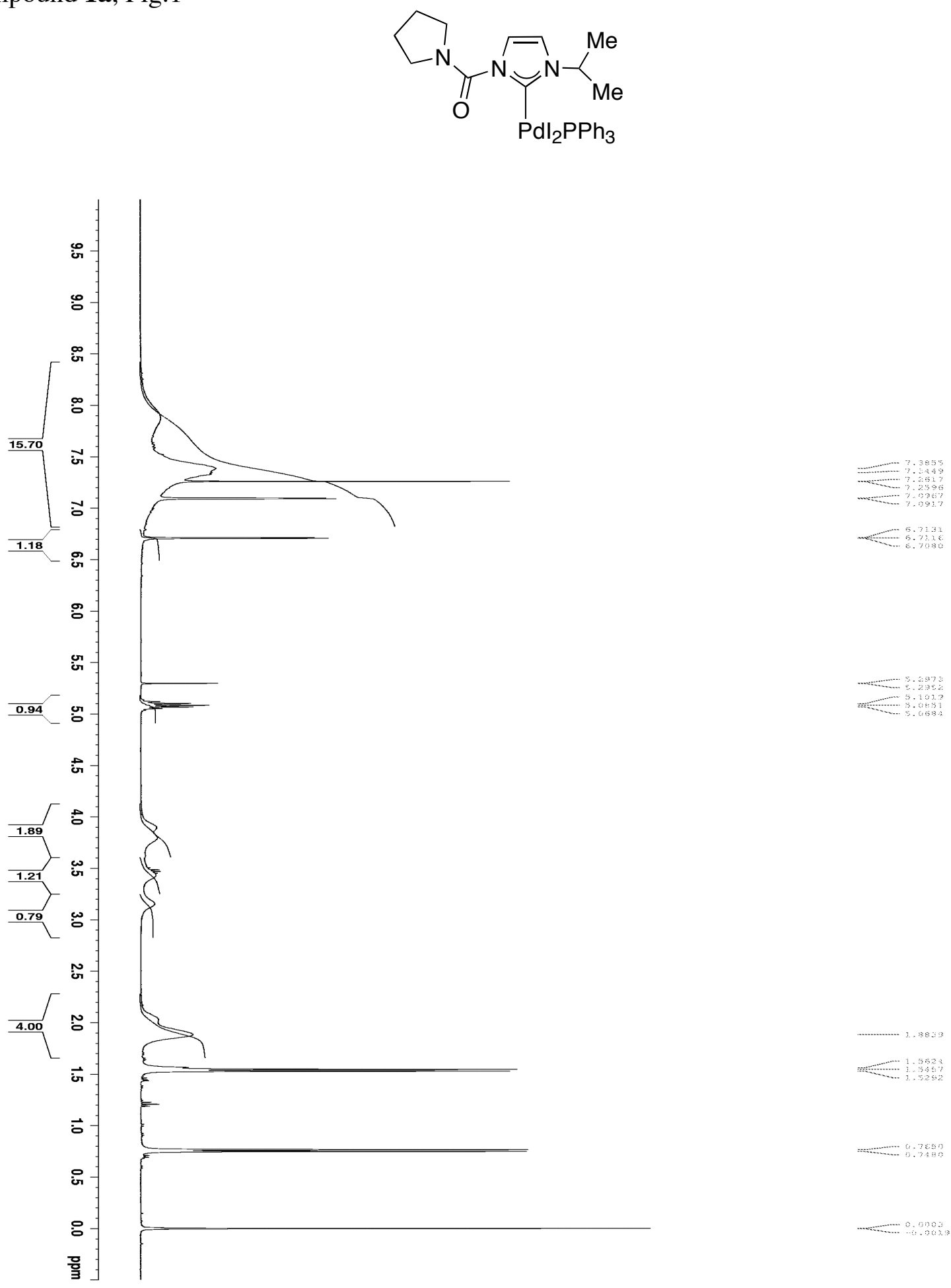

The pyrrolidine and phosphine signals are very broad. The stereochemistry of the complex still remains to be established. 


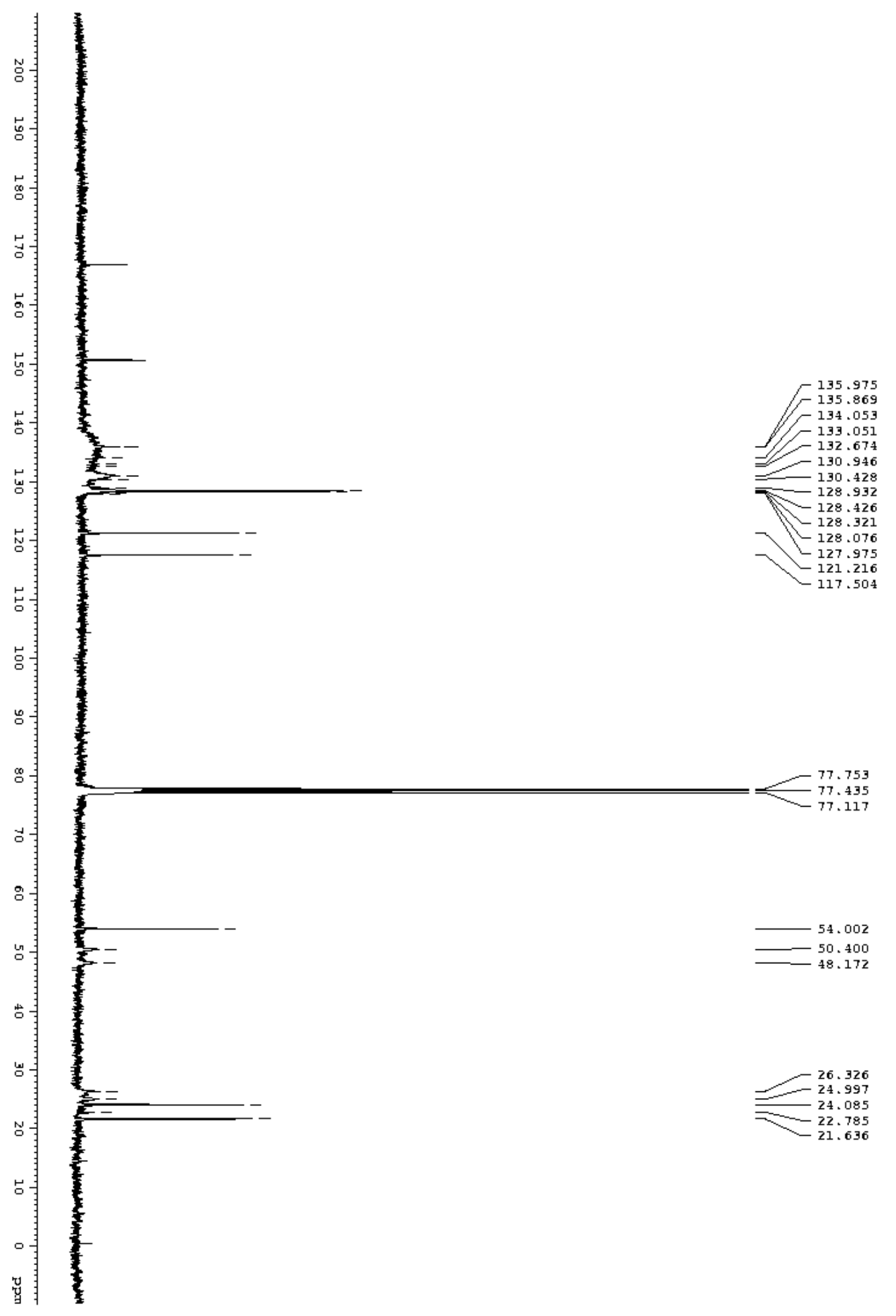


Compound 1b, Fig. 1
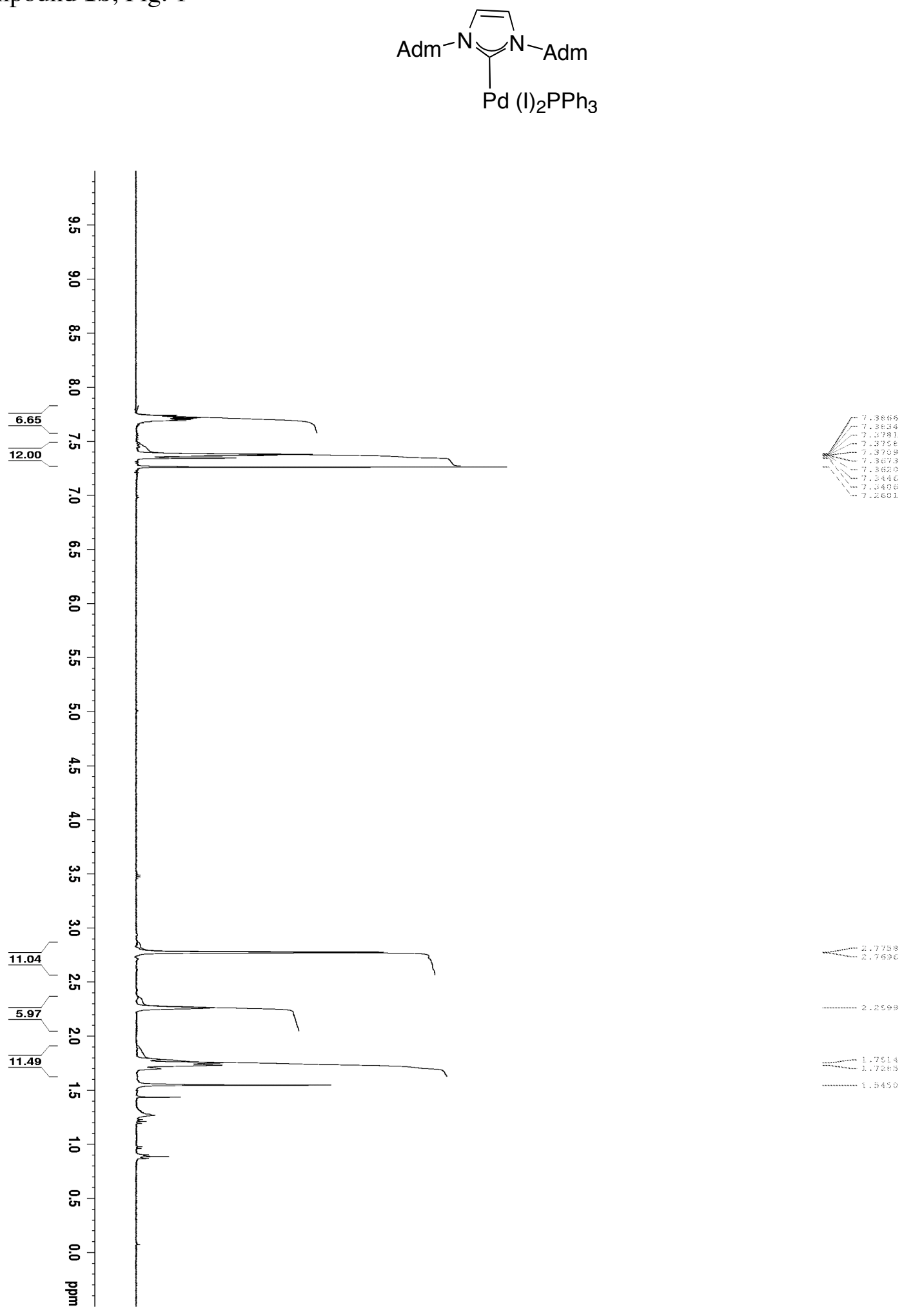

The stereochemistry of $\mathbf{1 b}$ has not yet been confirmed 


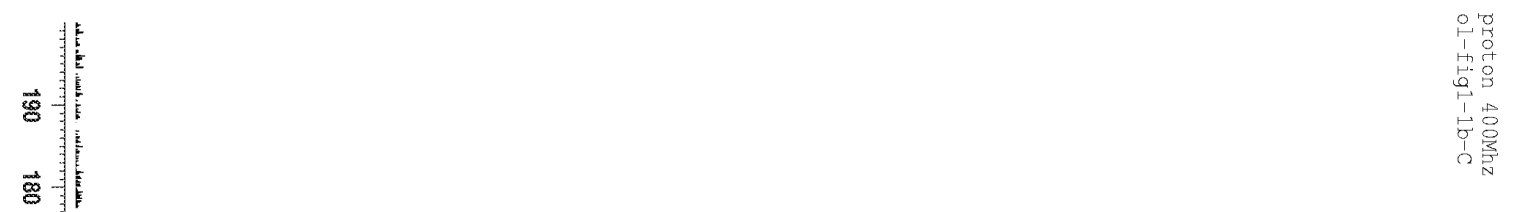

进
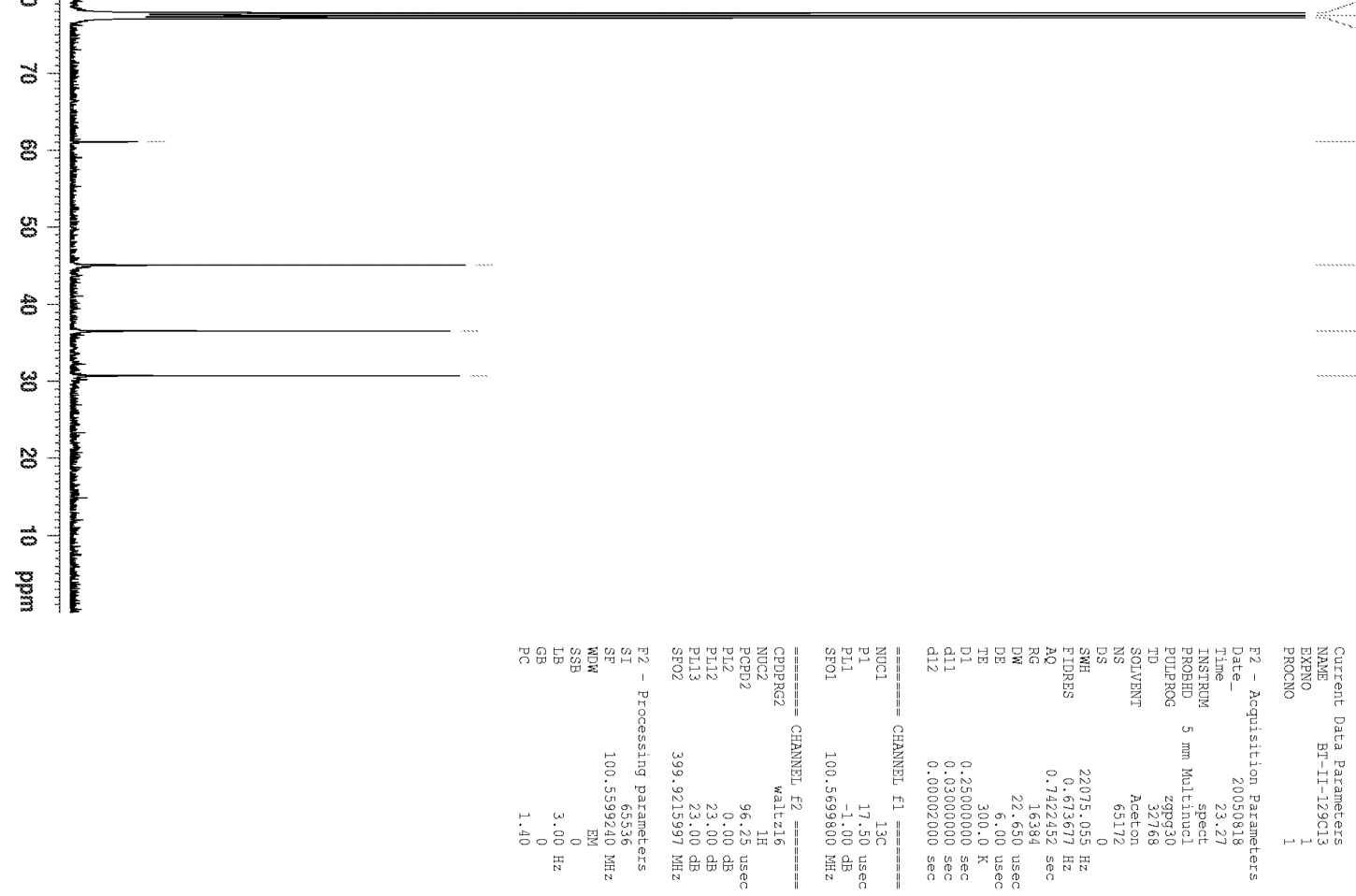
Compound 1c, Fig 1 (Proton and Carbon NMR spectra in $\mathrm{C}_{6} \mathrm{D}_{6}$ )<smiles></smiles>

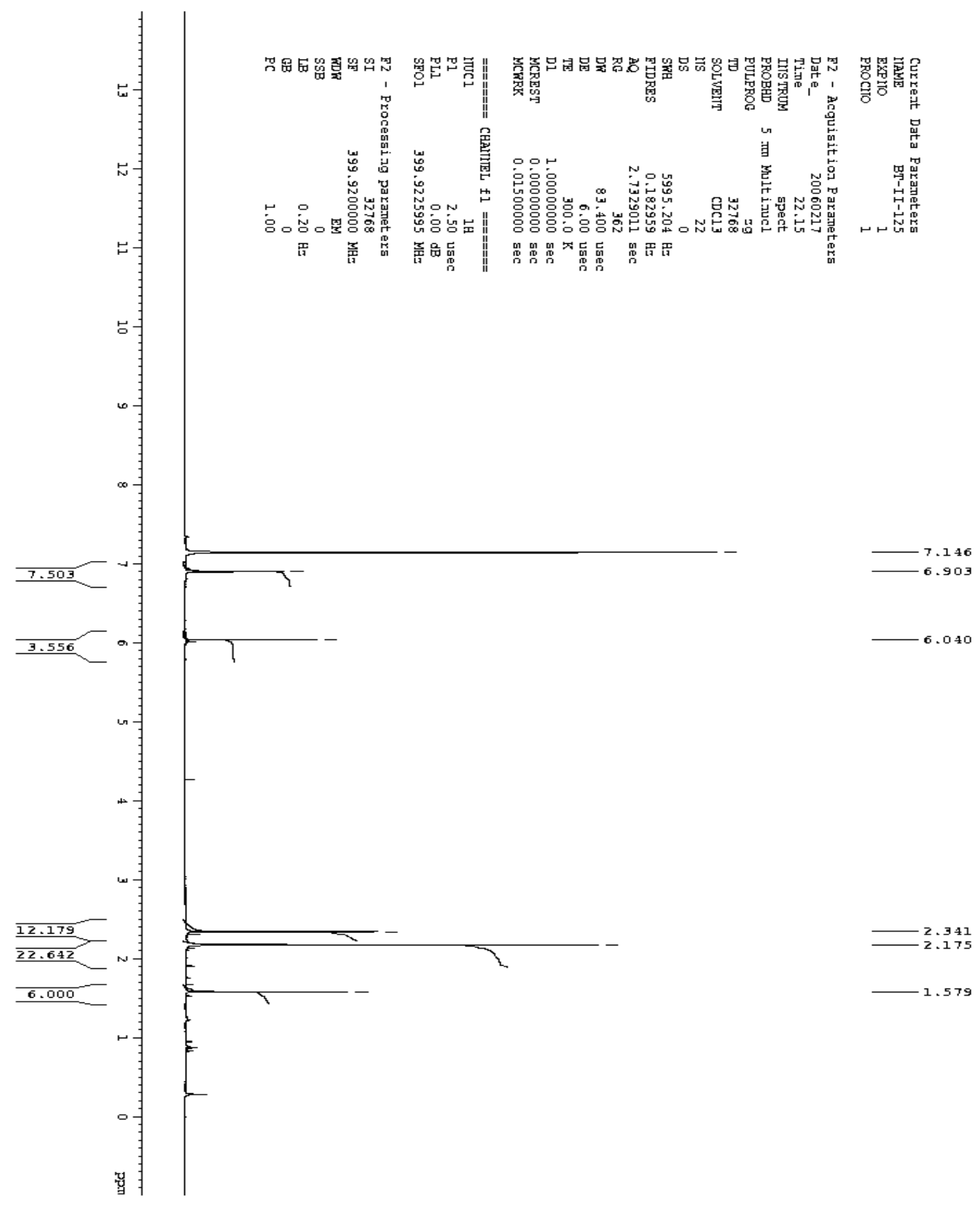




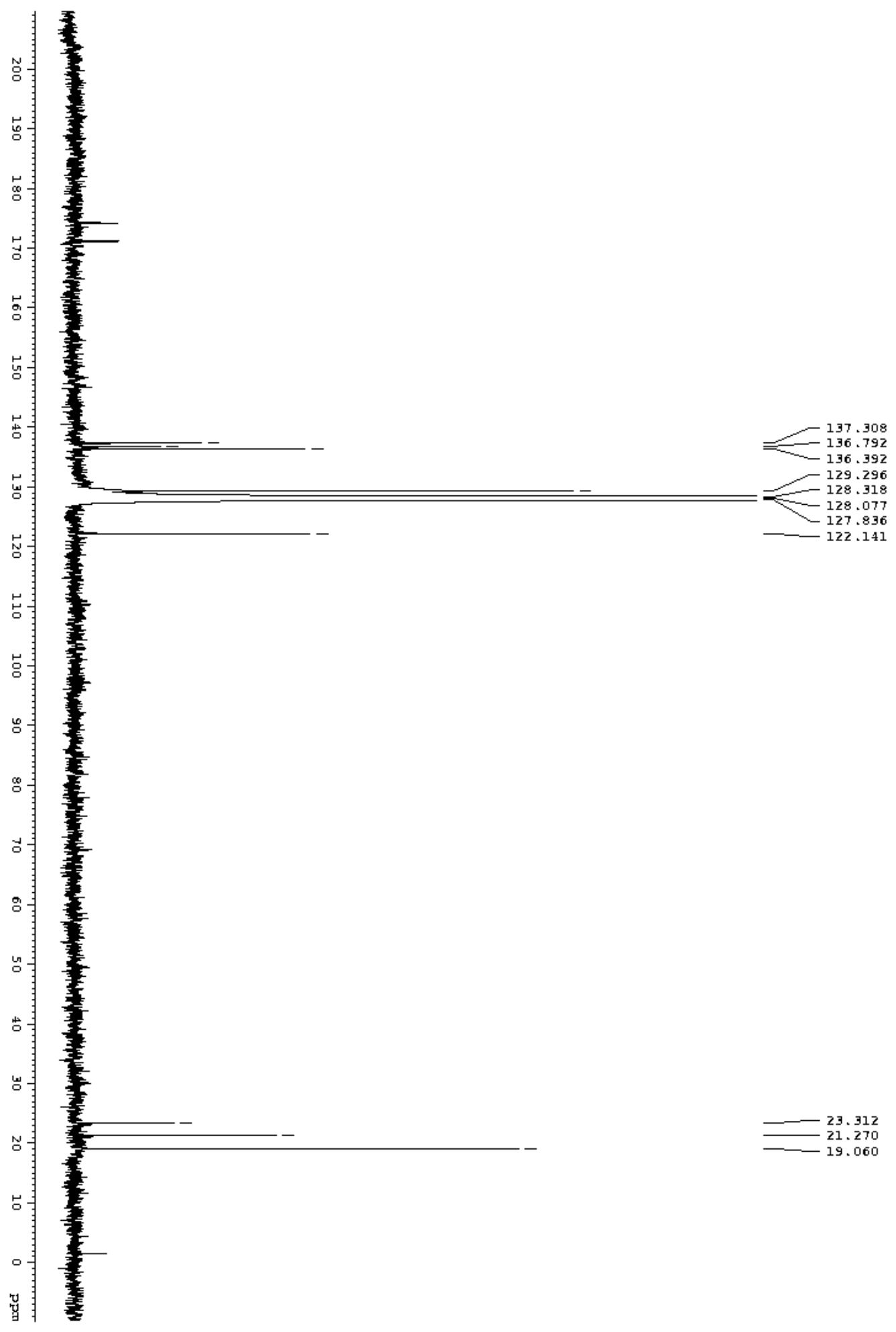


Compound 1c, Fig 1 (Proton and Carbon spectra in $\mathrm{CDCl}_{3}$ )

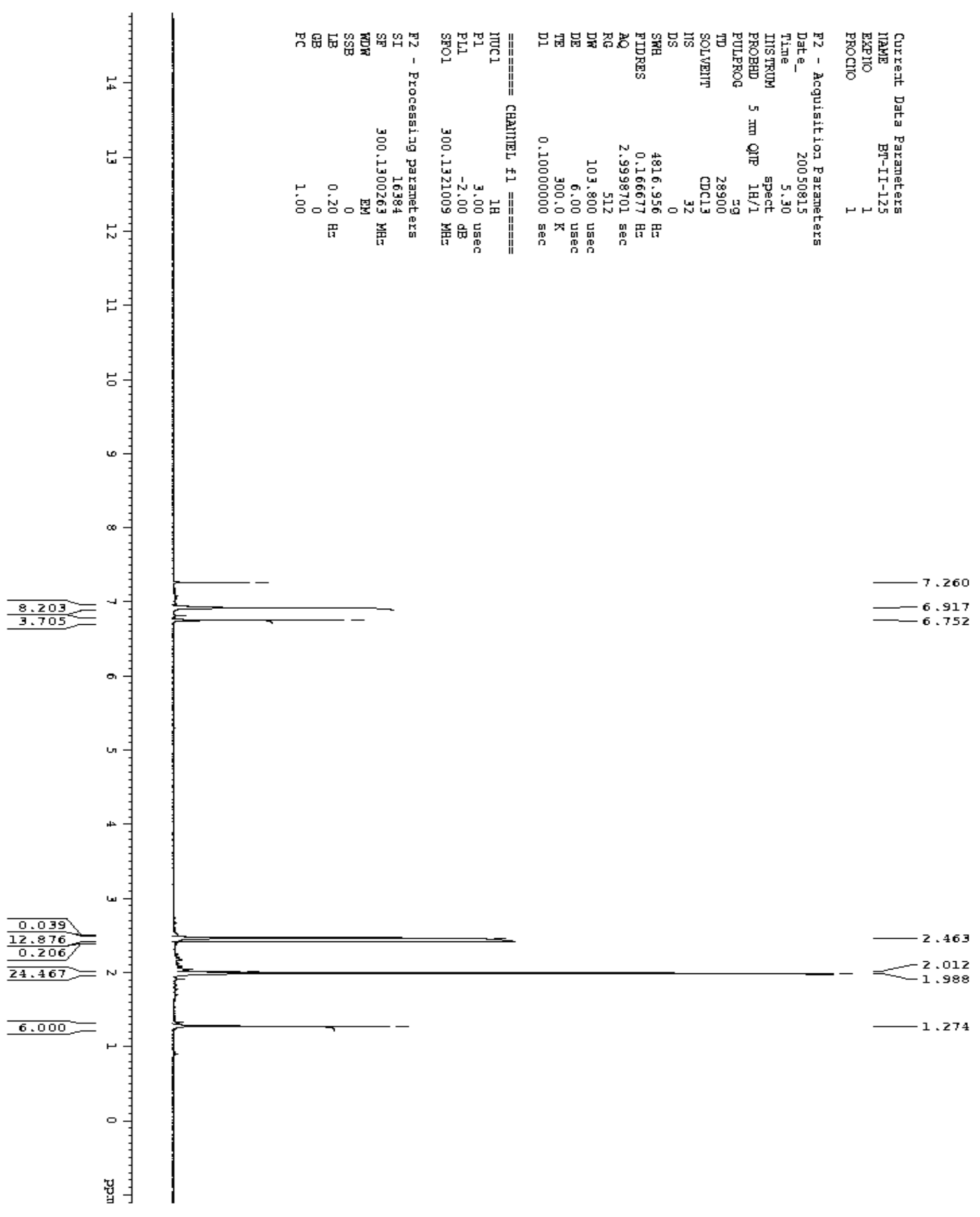




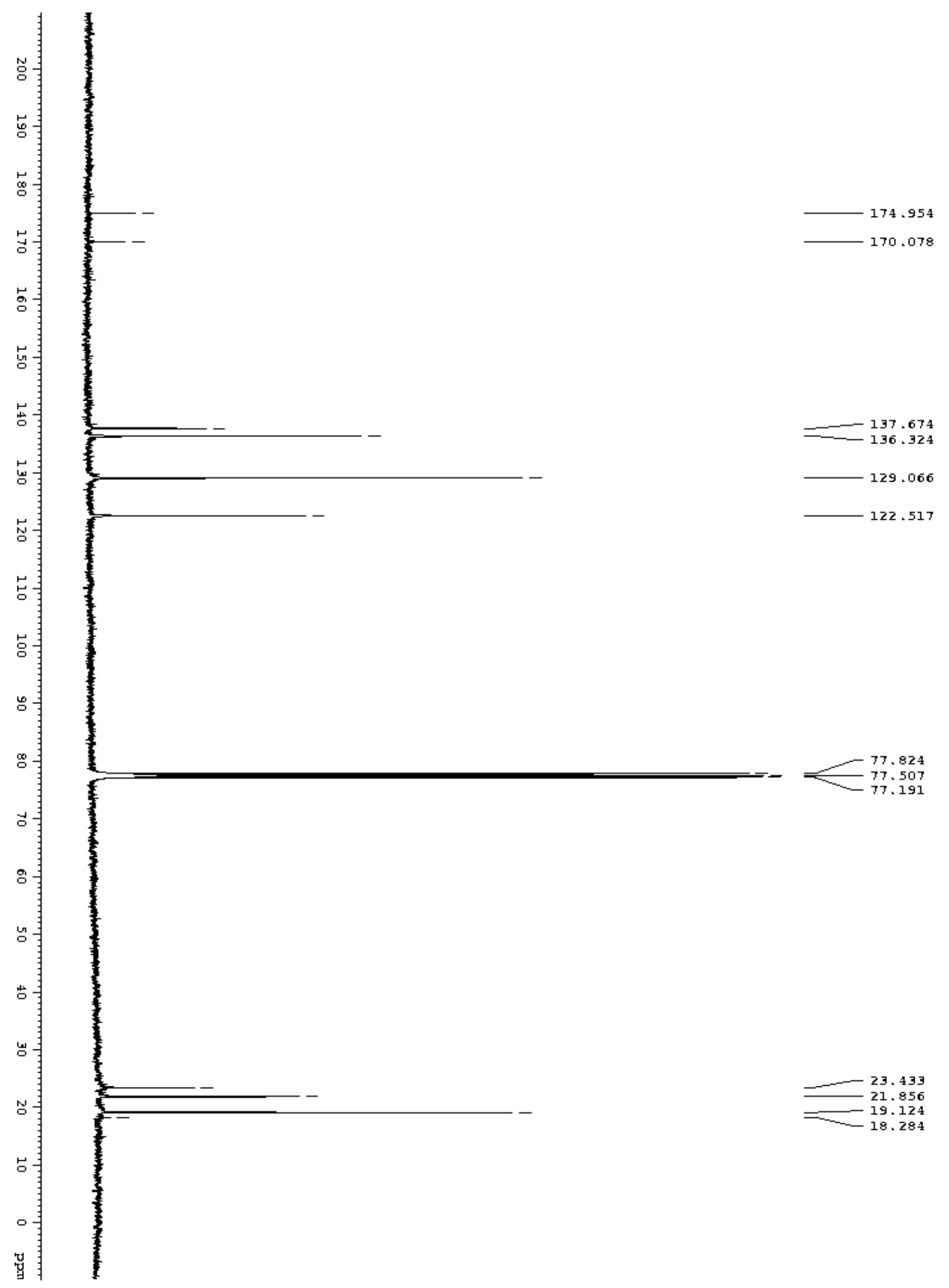




\section{Compound 1d, Fig 1}

$$
\prod_{\mathrm{Pd}(\mathrm{I})_{2} \mathrm{PPh}_{3}}
$$

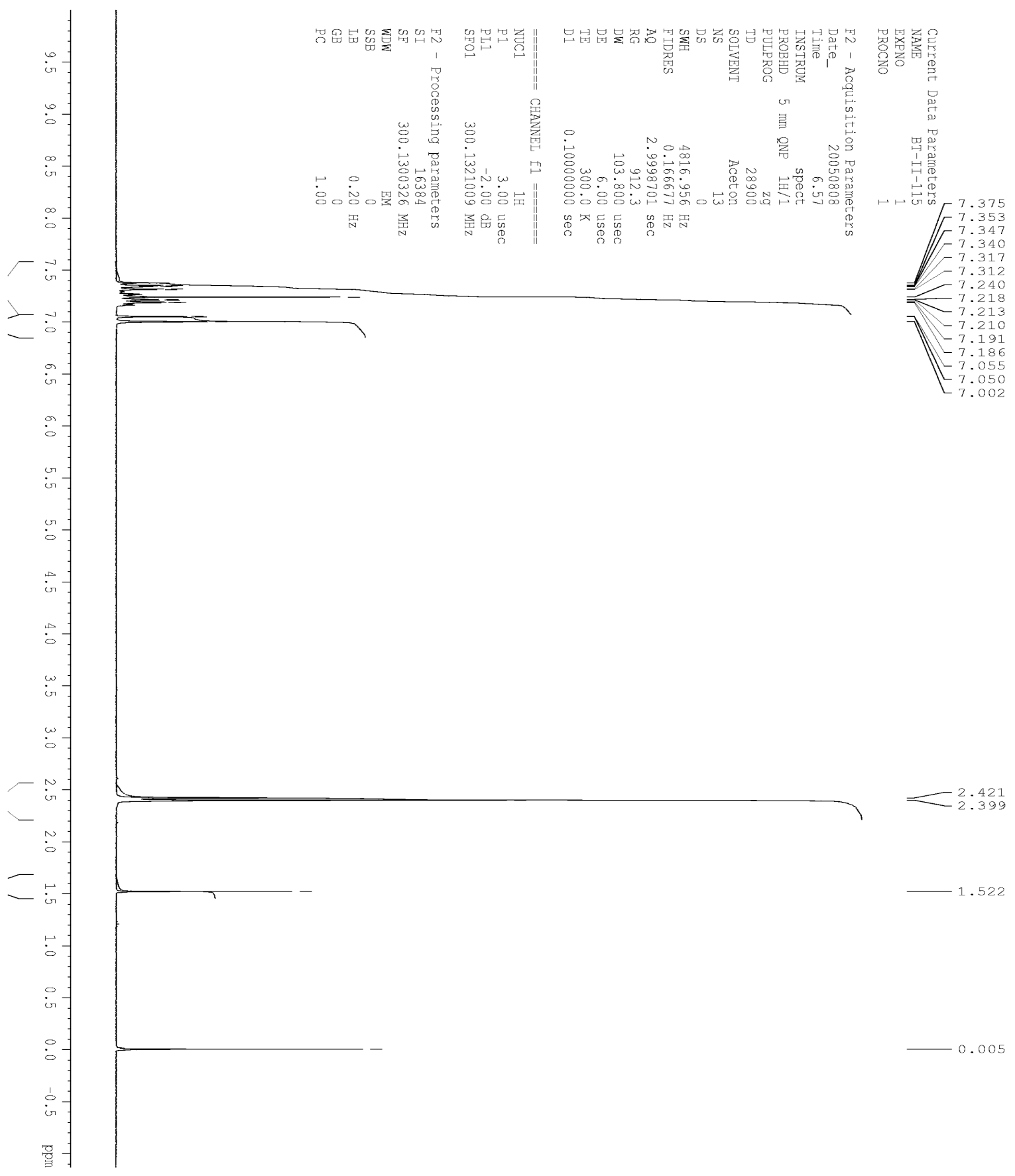



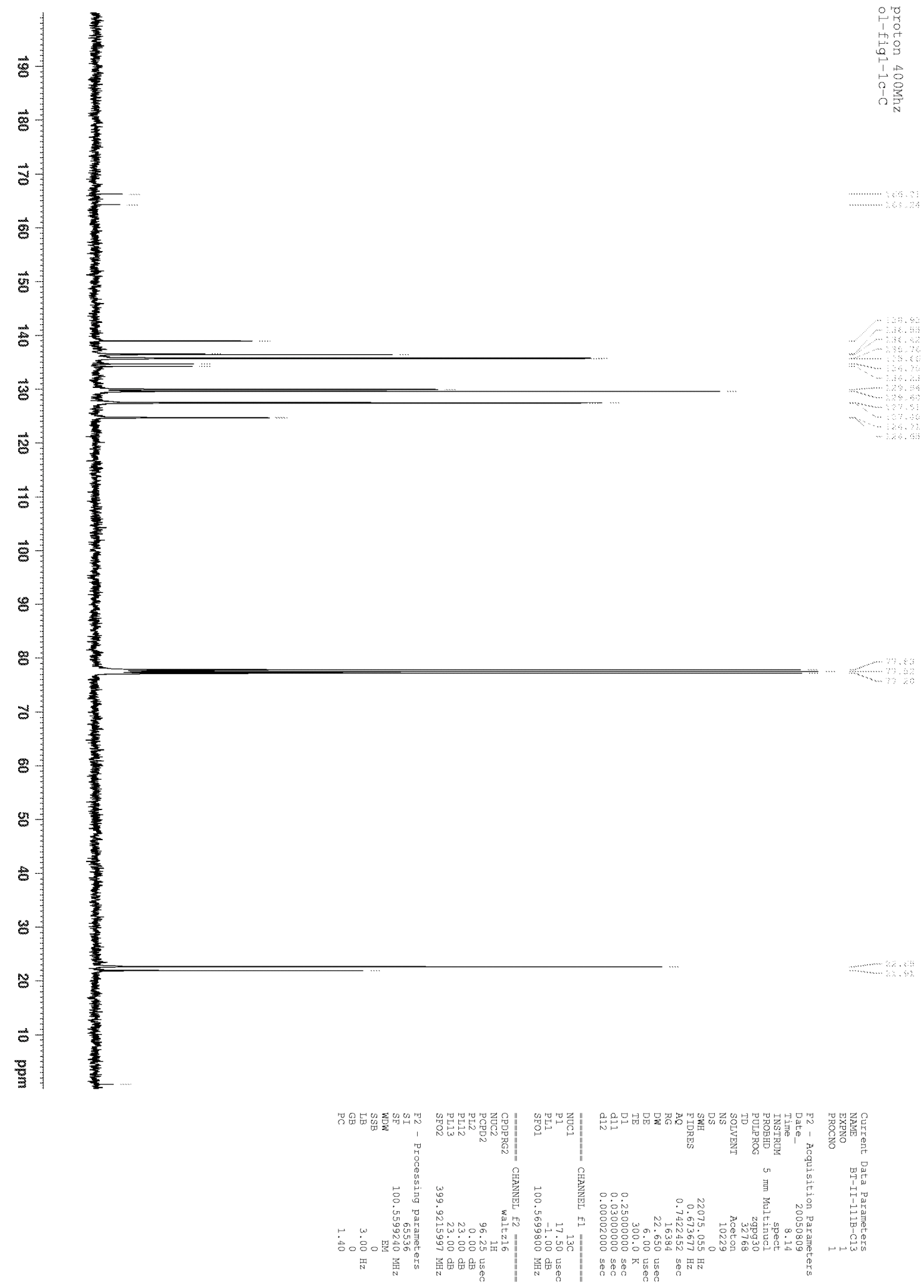
Table 1, entry 2. 2-Phenyl-1-[2-(trimethylsilyl)-ethoxymethyl]-indole<smiles></smiles>

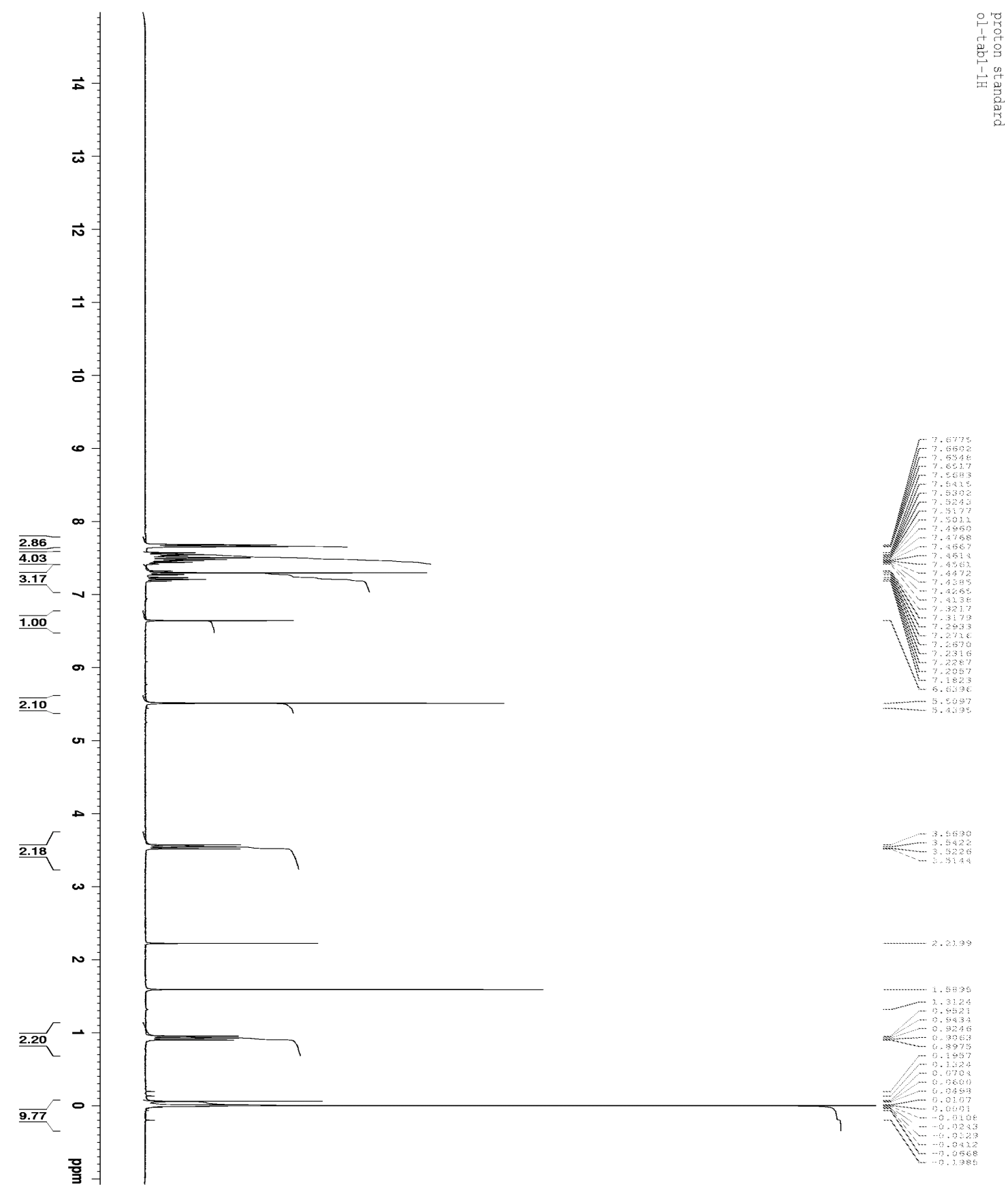




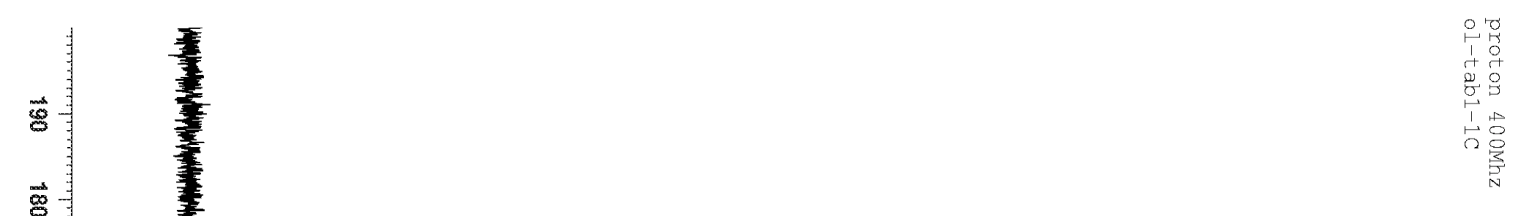

$\overrightarrow{\mathrm{s}}$

홍

g

$\overrightarrow{8}$

幽

$\vec{\Xi}$

$\overrightarrow{\vec{\theta}}$

है

8

8

$\approx$

8

8

8

s

8

$\stackrel{\circ}{\circ}$

쁄

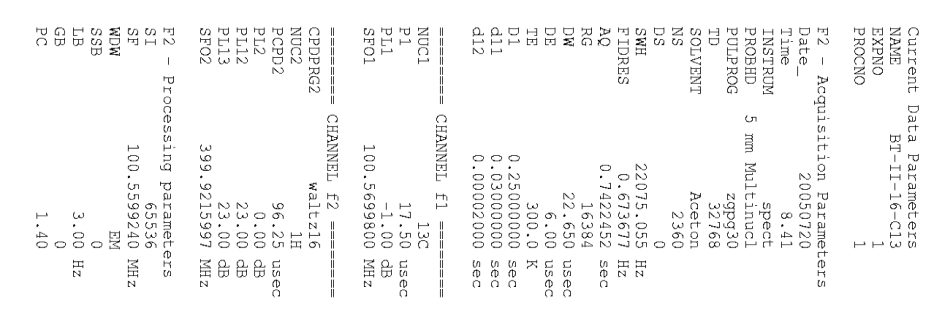


Table 1, Entry 3. 5-Cyano-2-Phenyl-1-[2-(trimethylsilyl)-ethoxymethyl]-indole<smiles></smiles>
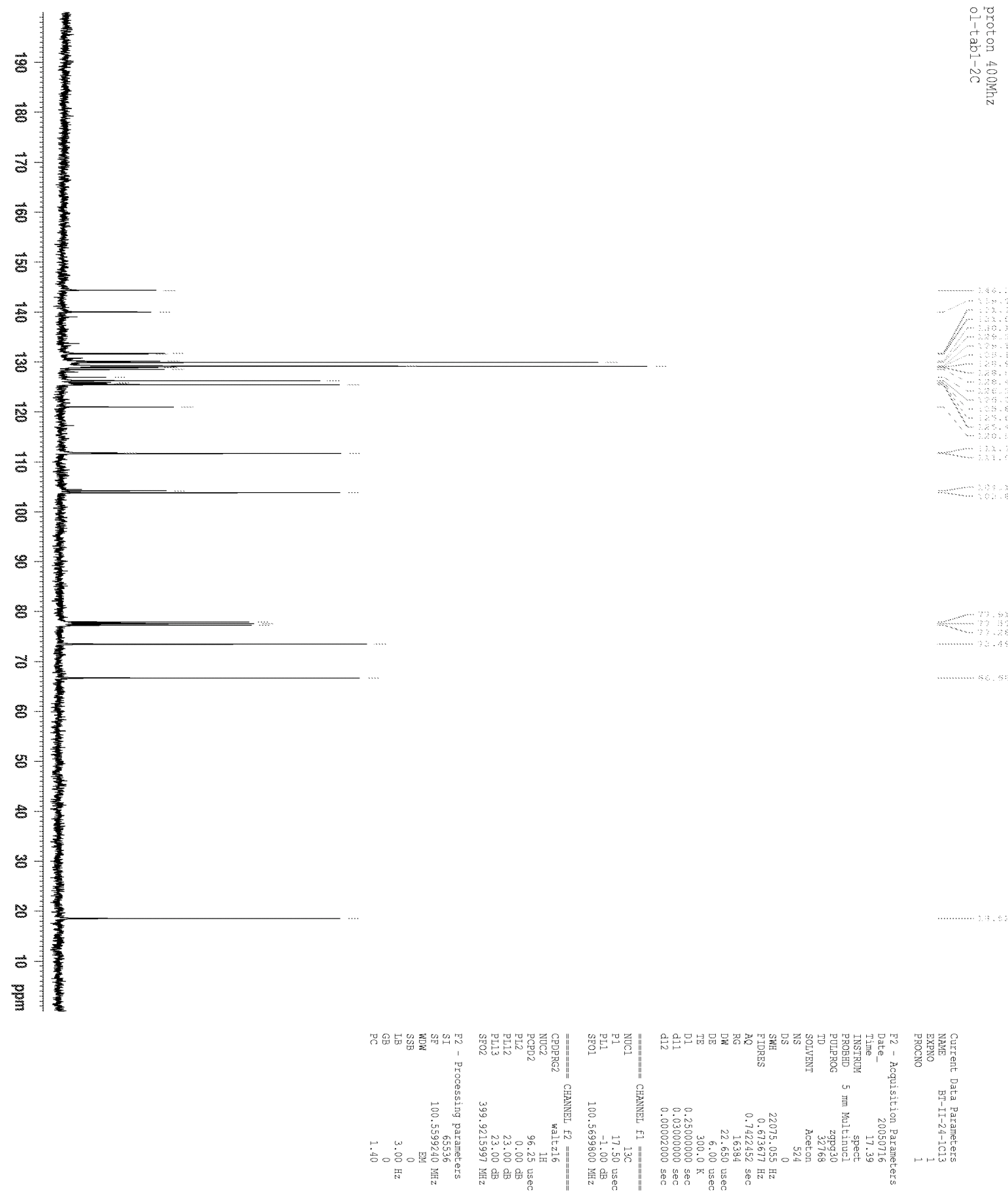
Table 1, entry 4. 5-Nitro-2-phenyl-1-[2-(trimethylsilyl)-ethoxymethyl]-indole $\mathrm{O}_{2} \mathrm{~N}$<smiles>[M]=Cn1c(-c2ccccc2)cc2cc(C)ccc21</smiles>

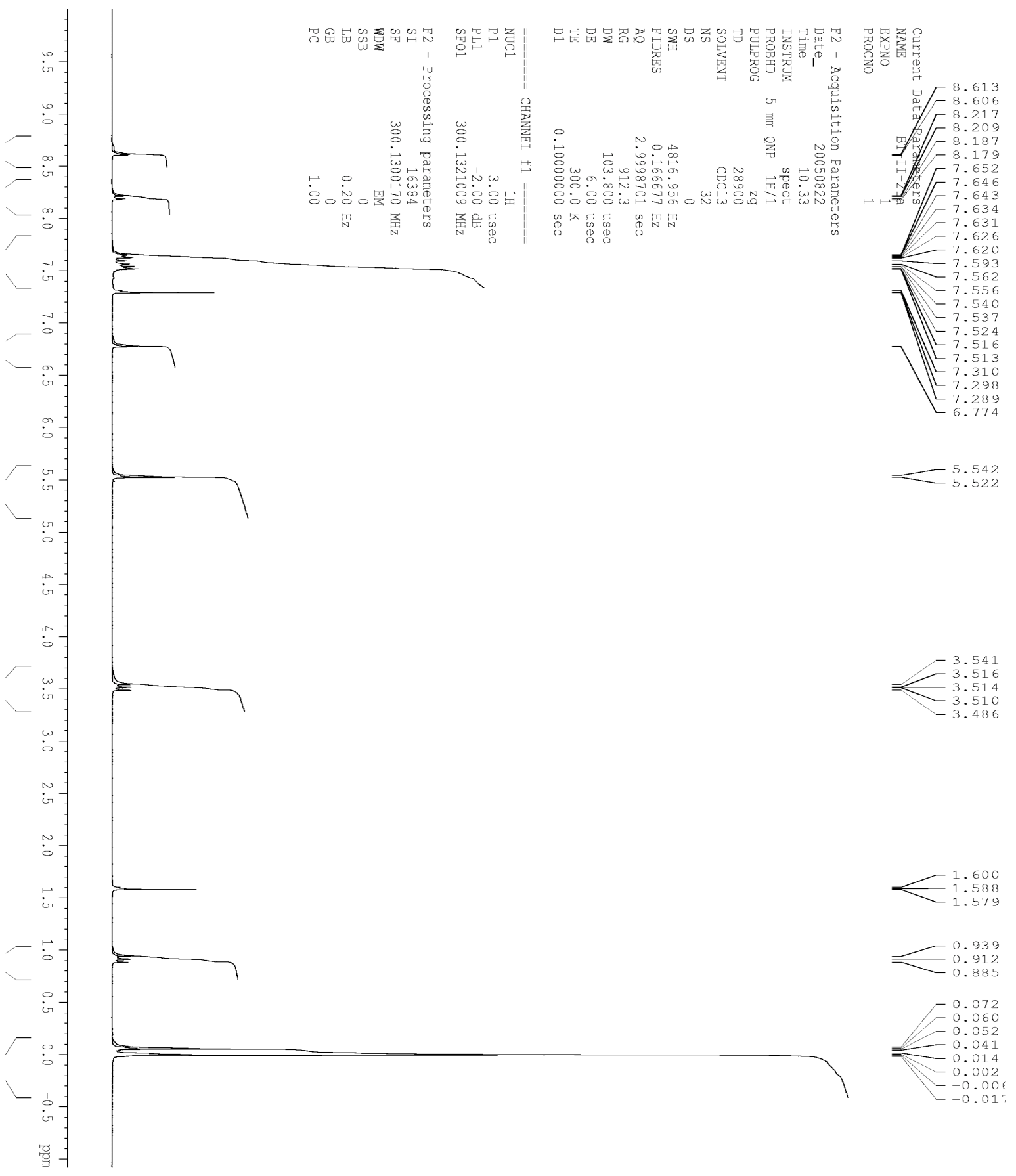


Table 1, entry 5 (2-o-Tolyl-1-[2-(trimethyl-silyl)-ethoxymethyl]-indole)<smiles>[M]#[SH+]n1c(-c2ccccc2C)cc2ccccc21</smiles>

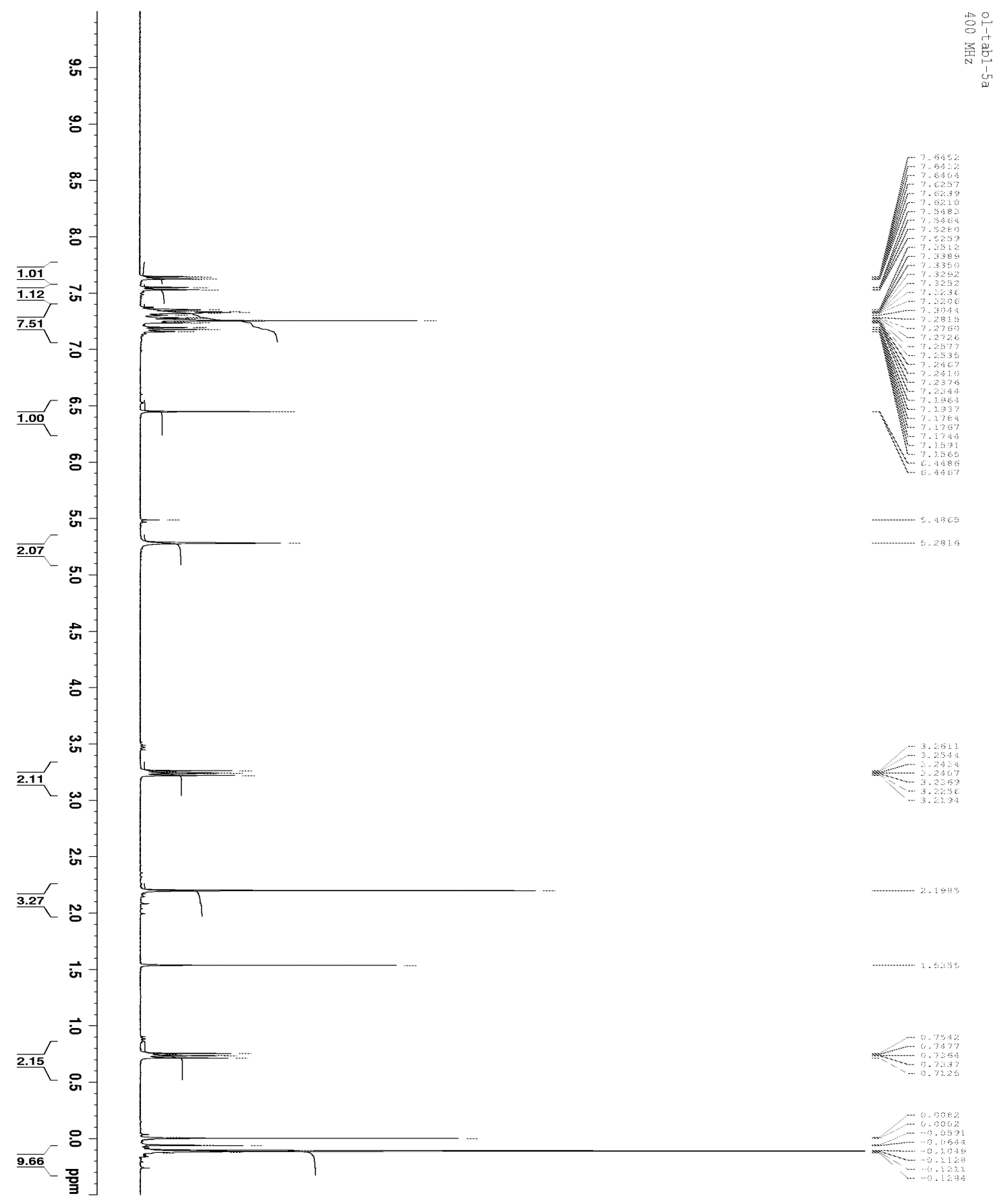


Table 1, entry 5 (3-o-Tolyl-1-[2-(trimethyl-silyl)-ethoxymethyl]-indole)<smiles></smiles>

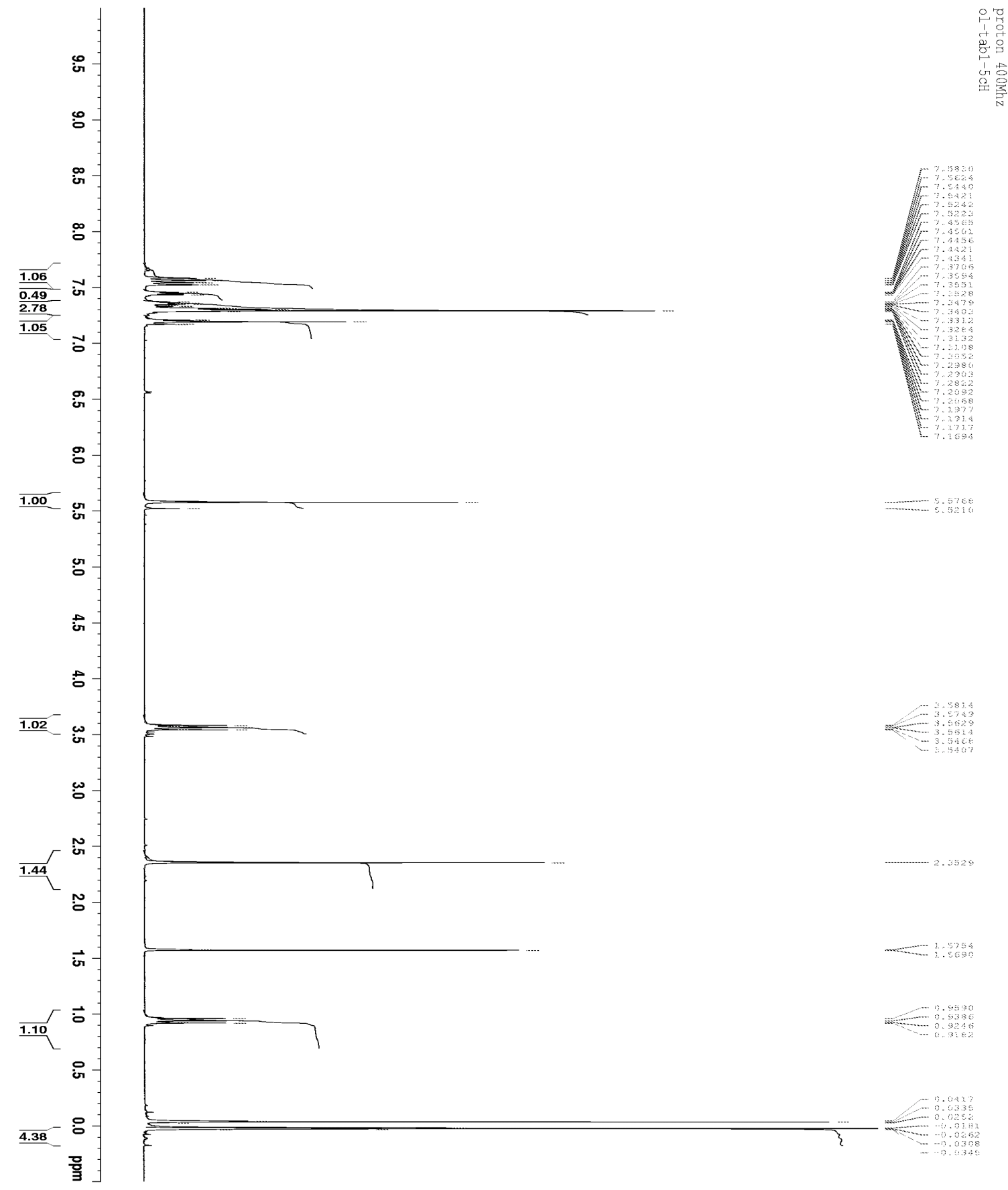




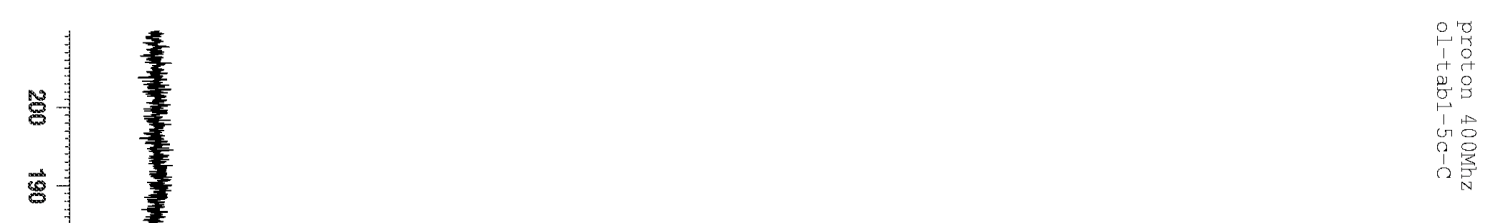

$\ddot{g}$

$\vec{d}$

$\overrightarrow{\mathrm{g}}$

$\overrightarrow{\mathrm{g}}$

咅

$\overrightarrow{\vec{g}}$

$\overrightarrow{3}$

ᄒे

$\vec{\Xi}$

$\because$

\&

$\approx$

용

gg

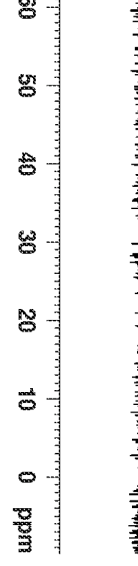

뜽

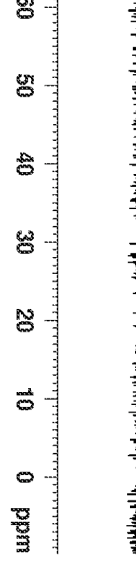

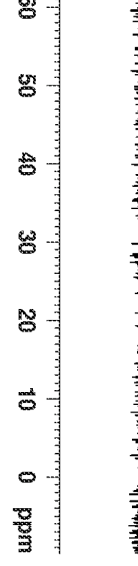

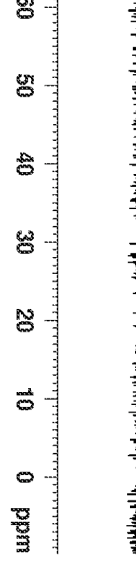

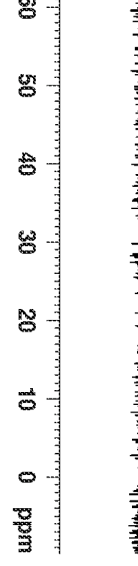

重

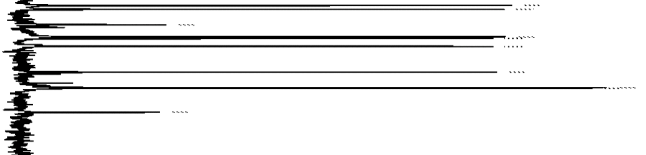

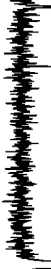
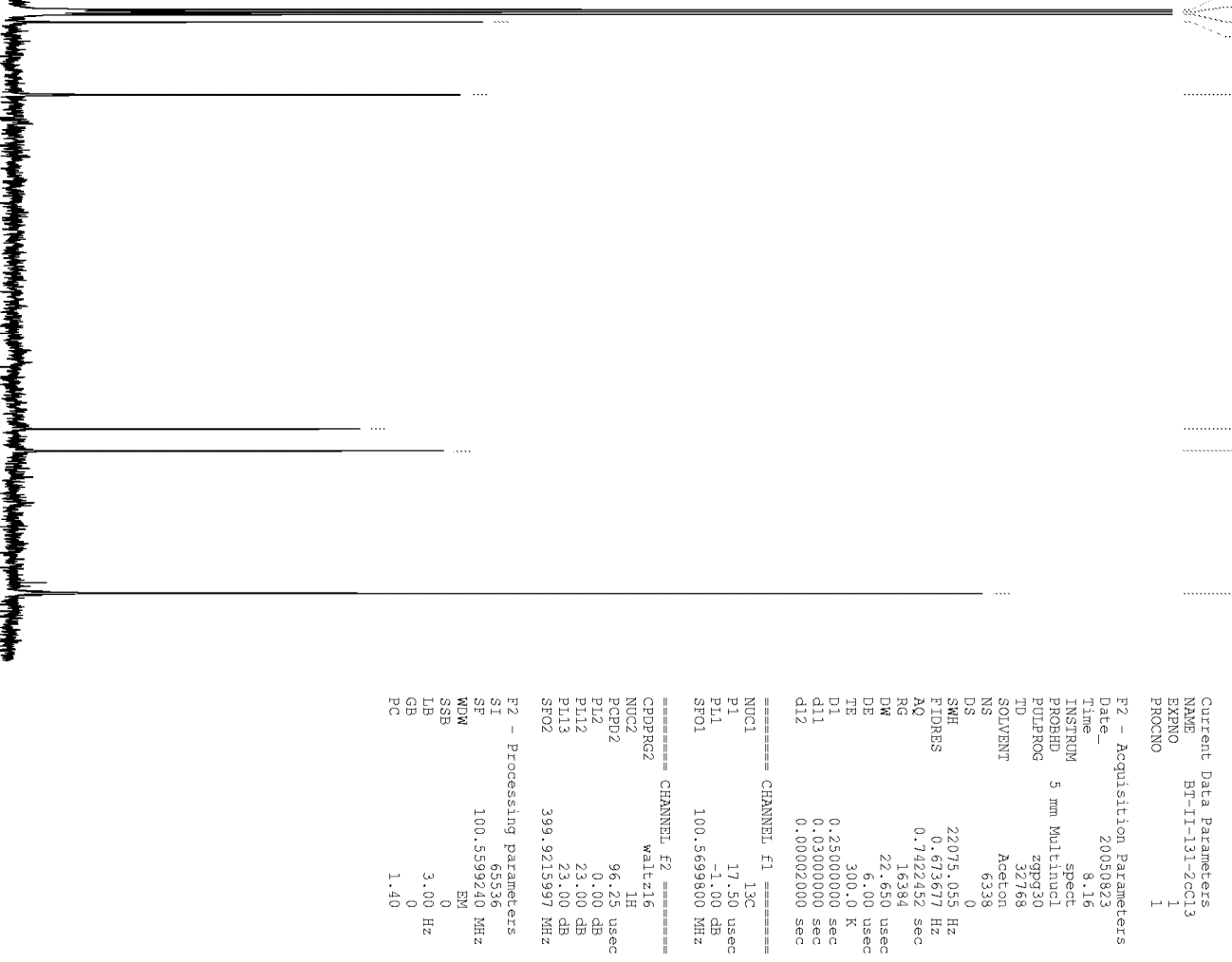
Table 1, entry 6. 2-Phenyl-1-[2-(trimethylsilyl)-ethoxymethyl]-indole-3-carboxylic acid methyl ester<smiles>CC(=O)c1c(-c2ccccc2)c2ccccc2n1-c1ccccc1</smiles>

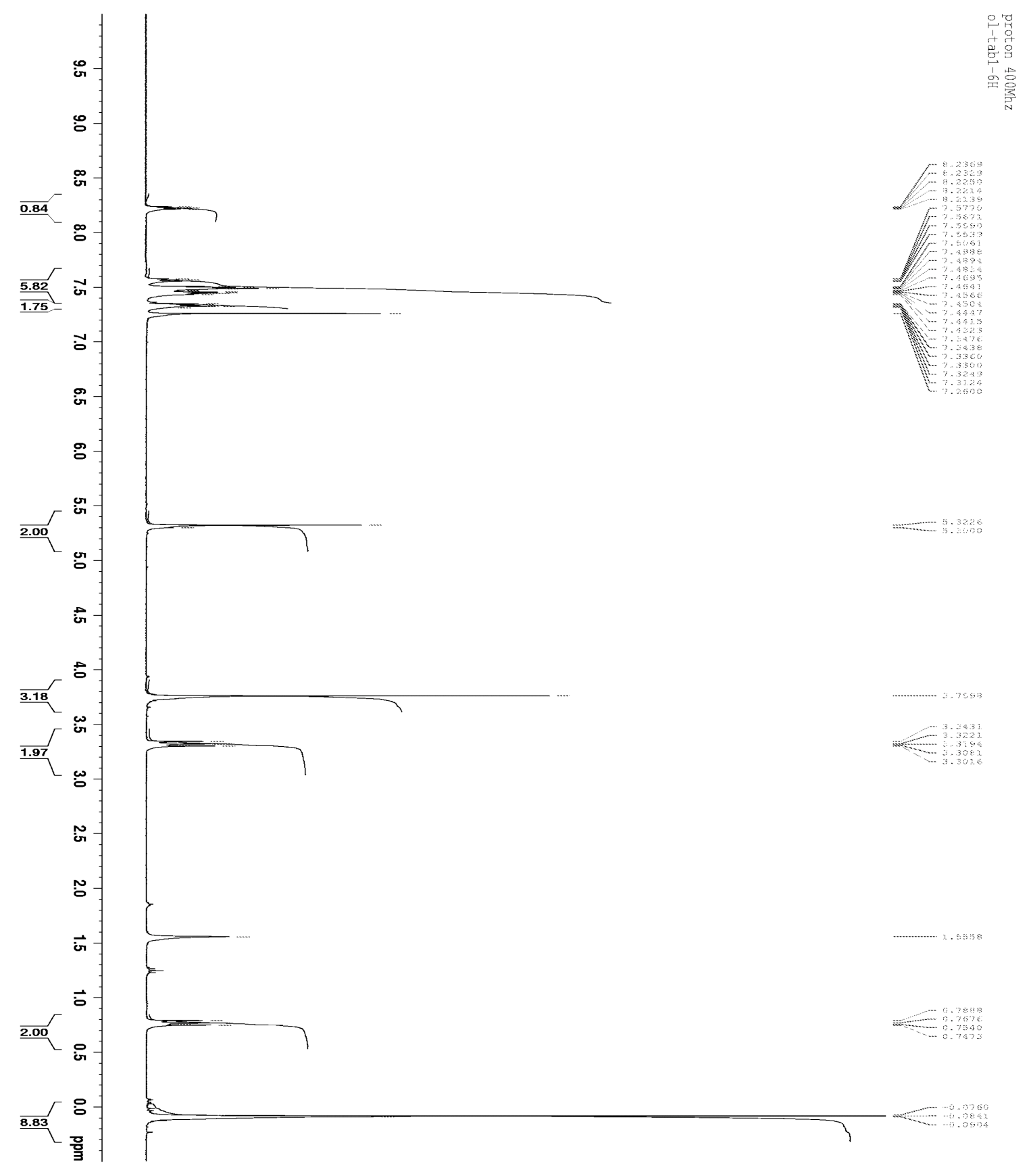


Table 1, entry 8. 5-Phenyl-1-[2-(trimethylsilyl)-ethoxymethyl)-pyrrole-2-carbonitrile.<smiles>N#[R15]n1c(C#N)ccc1-c1ccccc1</smiles>

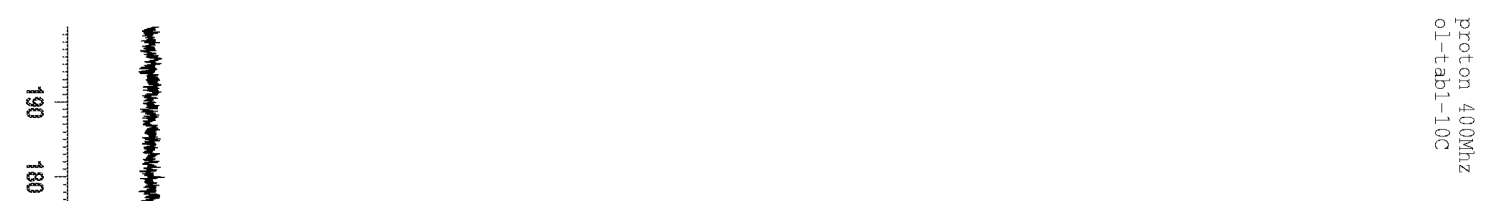

쁄

裏

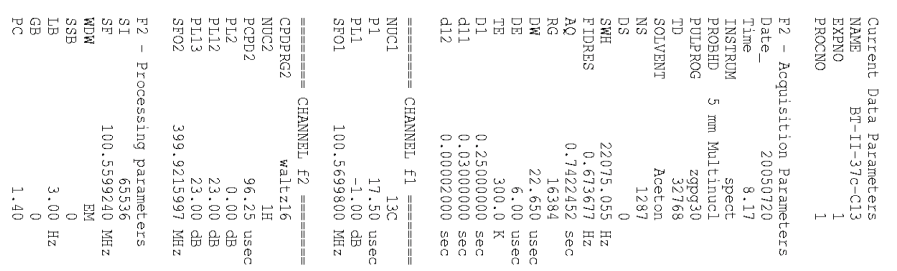


Table 1, entry 9. 5-Pyridin-3-yl-1-[2-(trimethylsilyl)-ethoxymethyl]-pyrrole-2-carbonitrile<smiles></smiles>

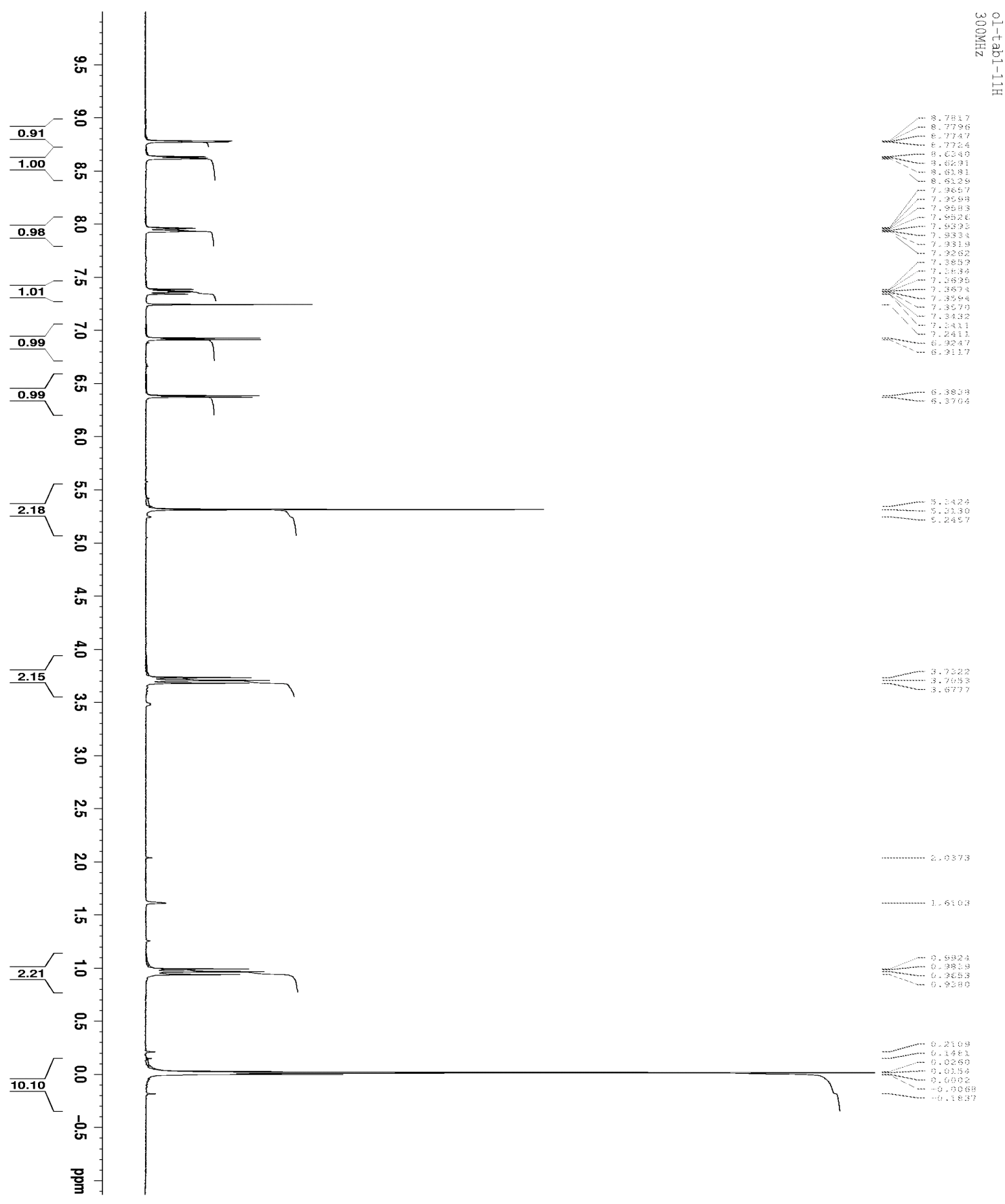




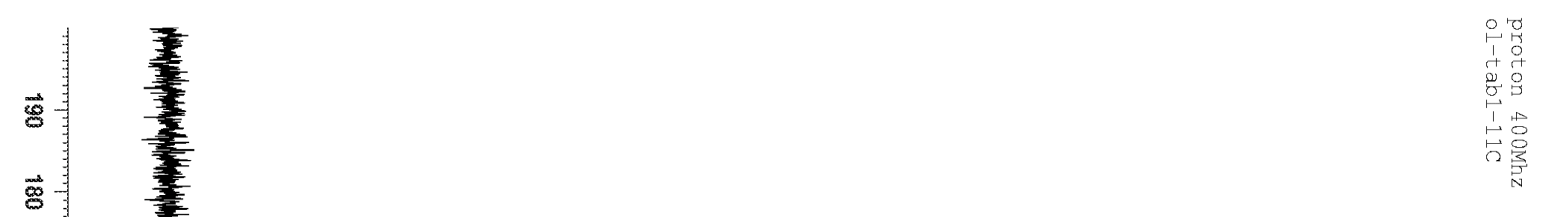

峛

$\overrightarrow{\mathrm{s}}$

$\stackrel{s}{\sigma}$

$\overrightarrow{\mathrm{s}}$

8

g

o

8

s

5

$\mathscr{E}$

s

b

$\circ$

్ㅗㅇ

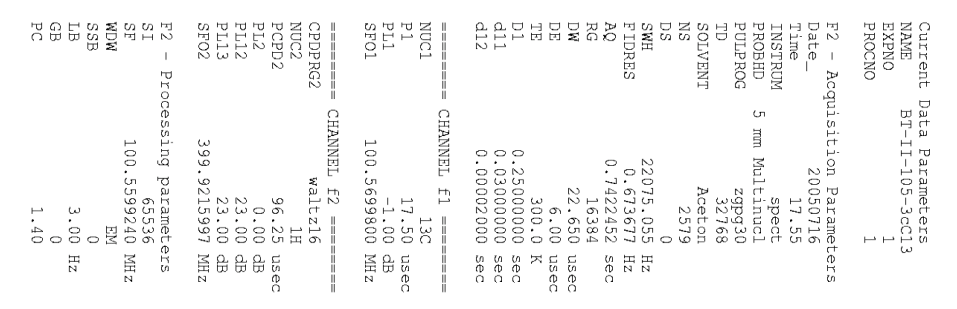


Table 1, Entry 10. 1-\{2,5-Diphenyl-1-[2-(trimethyl-silyl)-ethoxymethyl]-pyrrol-3-yl\}-ethanone

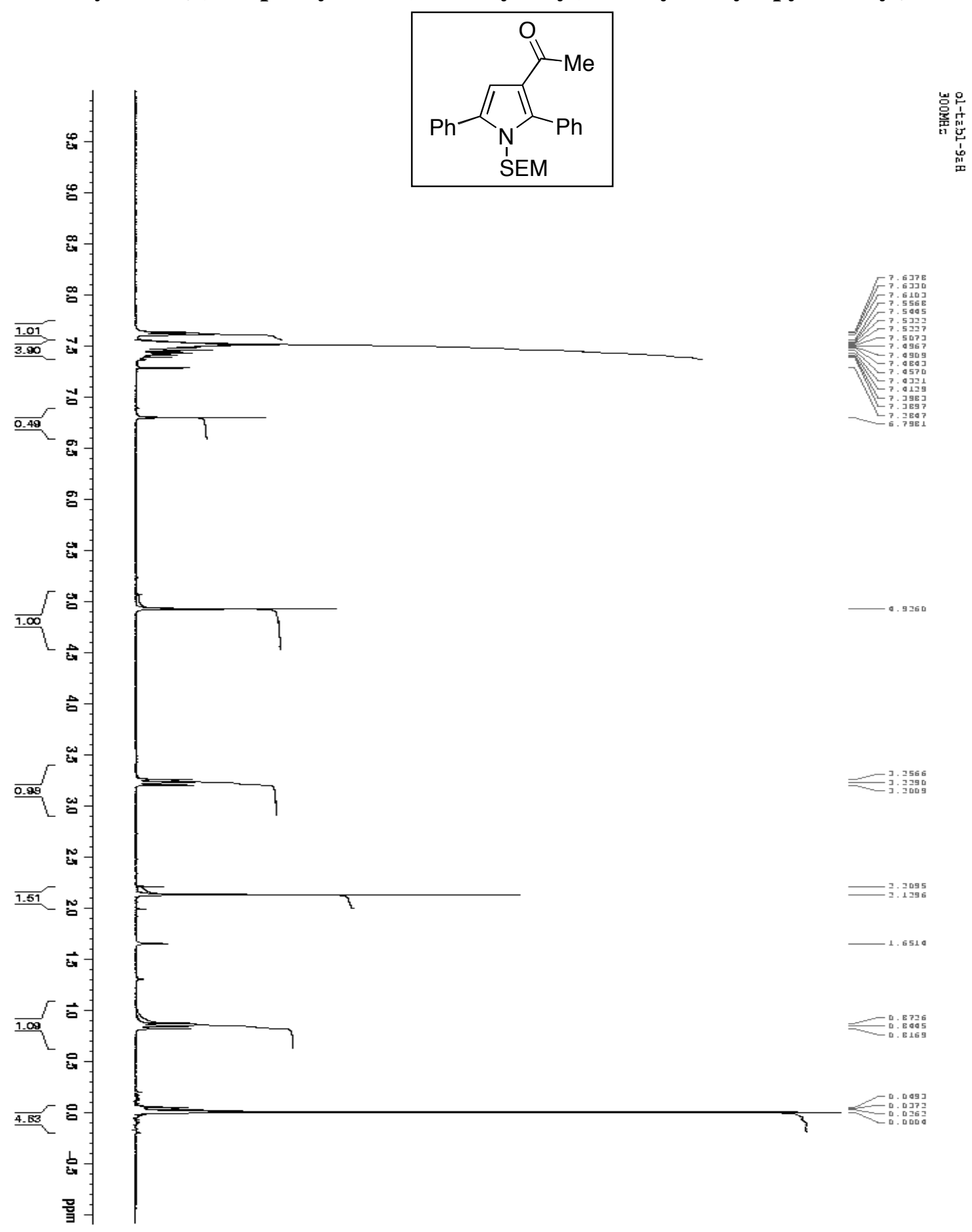



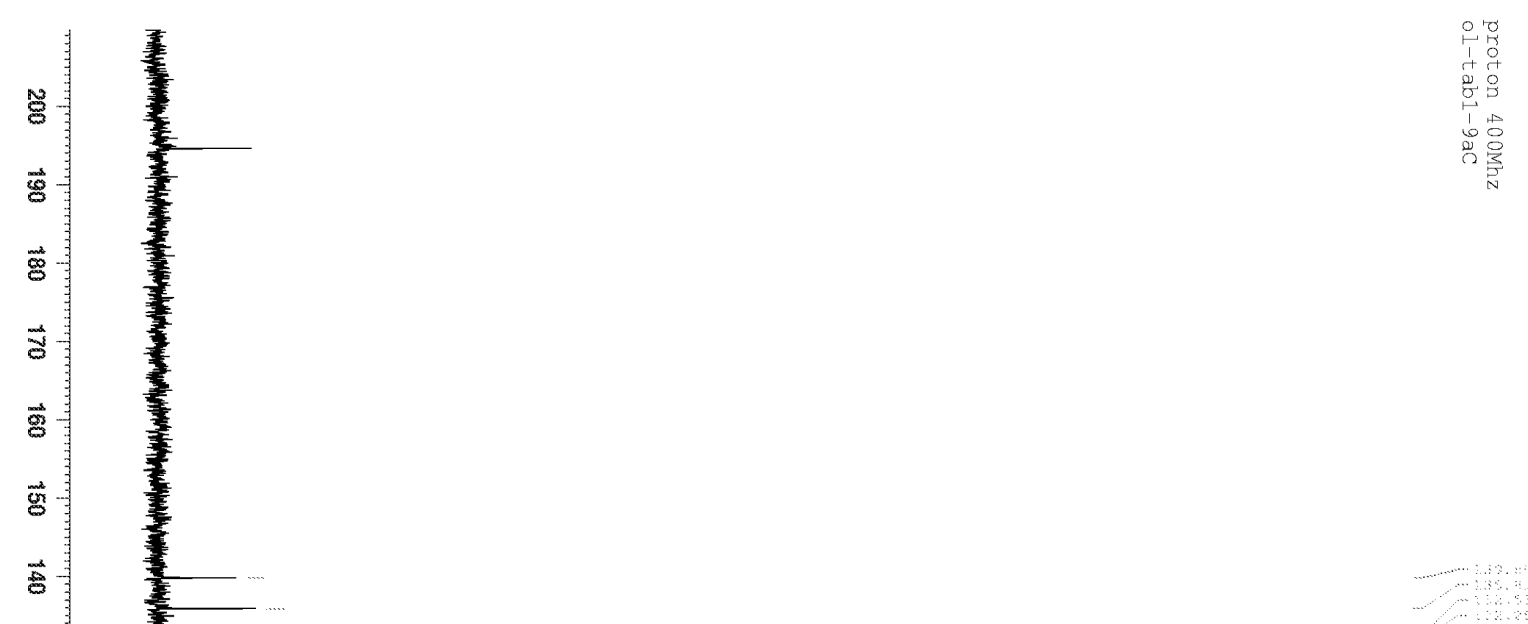

馬

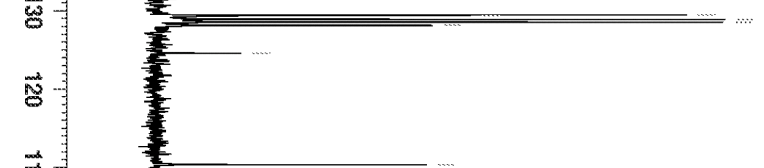

$\overrightarrow{\vec{b}}$

$\overrightarrow{8}$

8

\&

$\approx$

용

s

8

8

$\stackrel{8}{8}$

$\vec{\sigma}$

흘

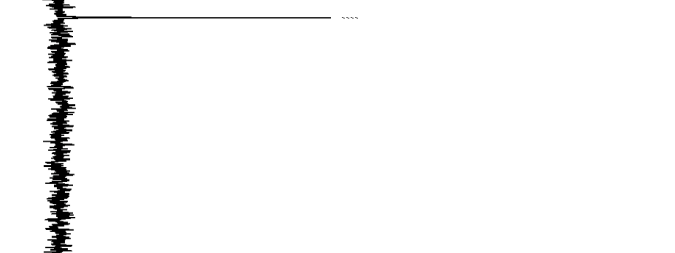


Table 1, entry 10. 1-\{2-phenyl-1-[2-(trimethyl-silyl)-ethoxymethyl]-pyrrol-3-yl\}-ethanone

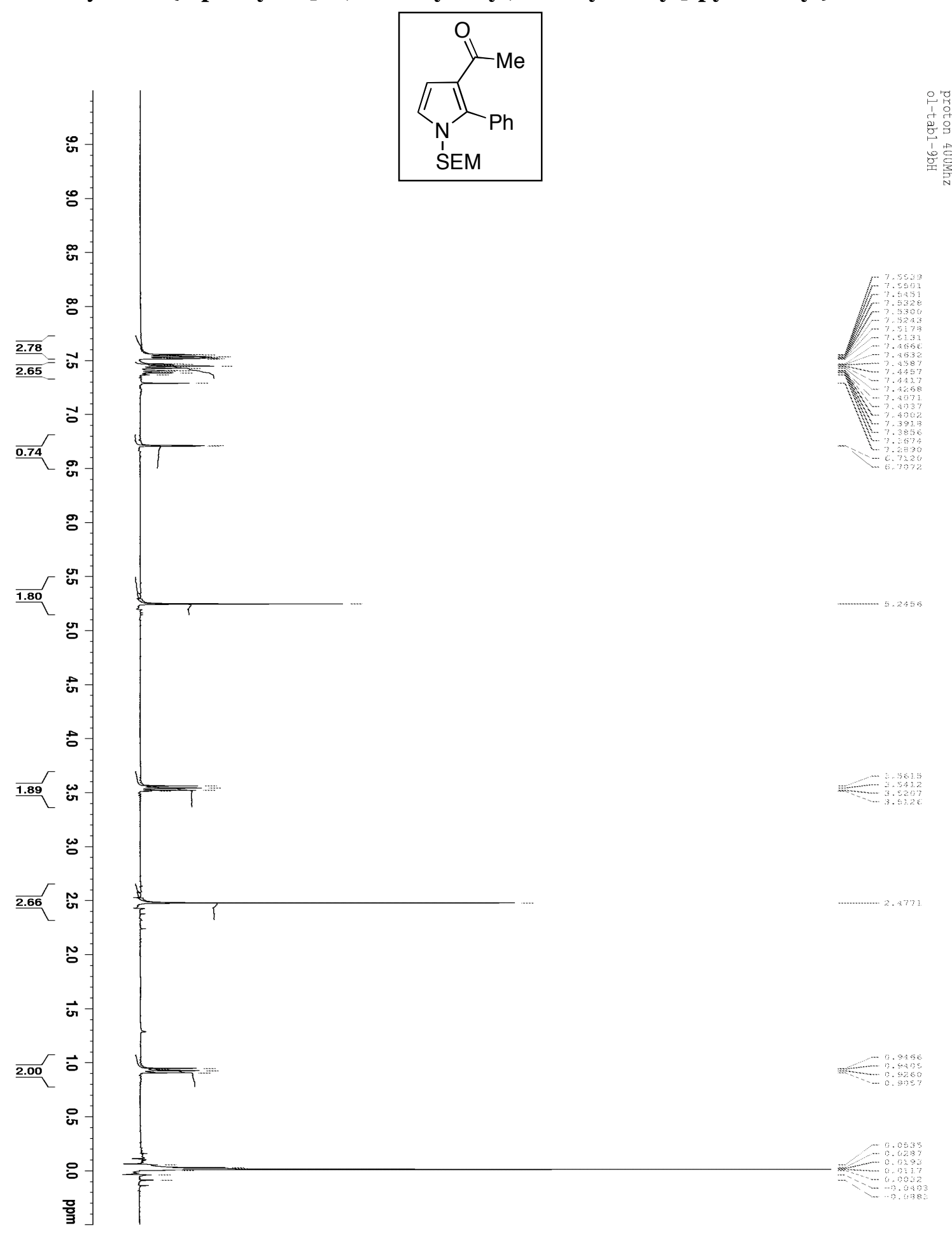




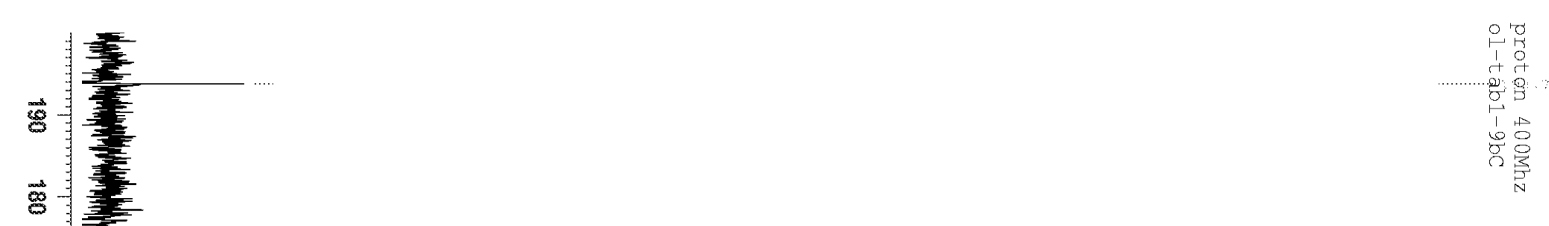

$\overrightarrow{8}$

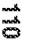

횽

8

:

o

8

용

\&

\&

8

के

0

뽘
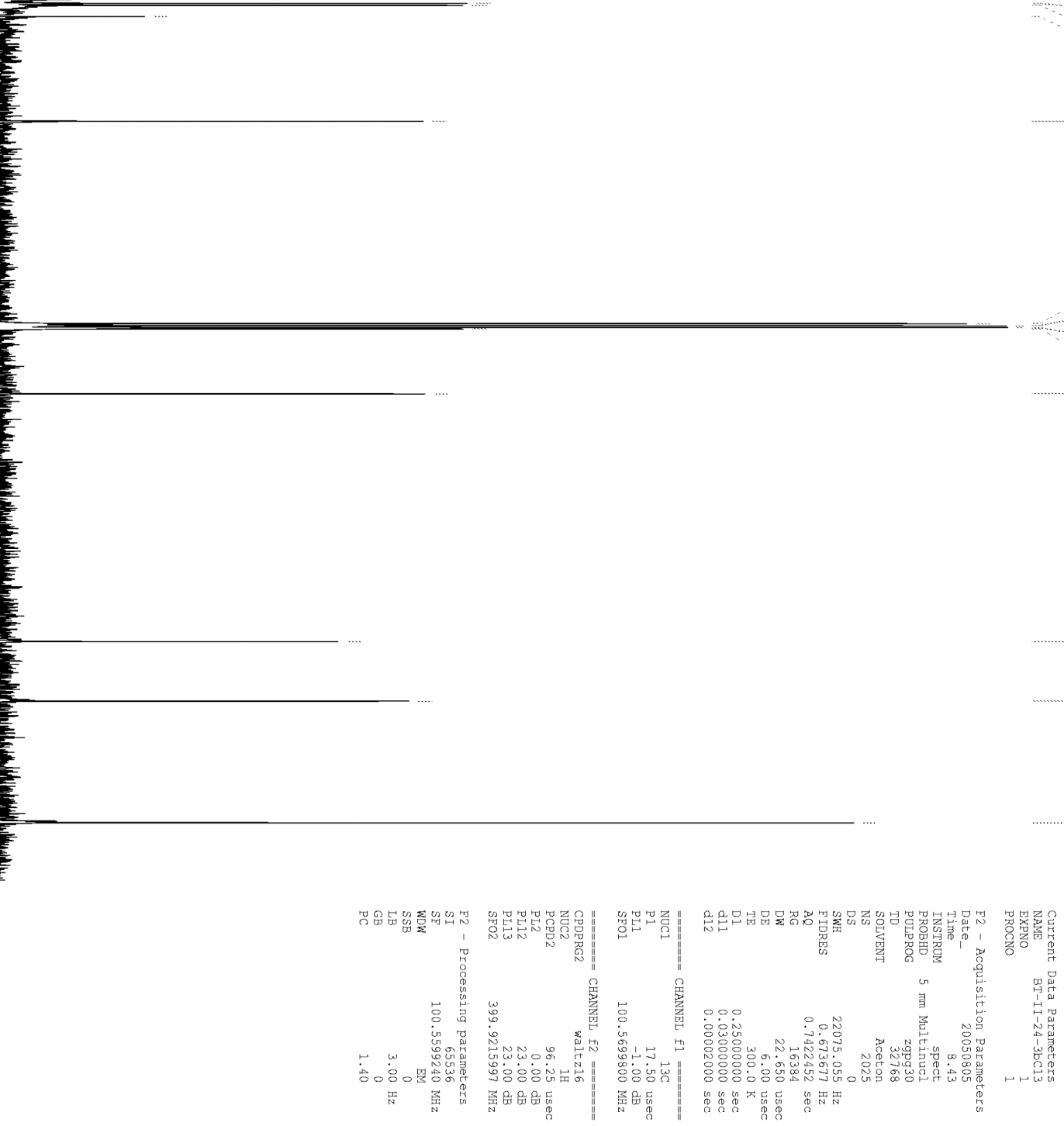
Table 2, entry 1. 2-Phenyl-1-[2-(trimethylsilyl)-ethoxymethyl]-7-azaindole<smiles>[M]#Cn1c(-c2ccccc2)cc2cccnc21</smiles>

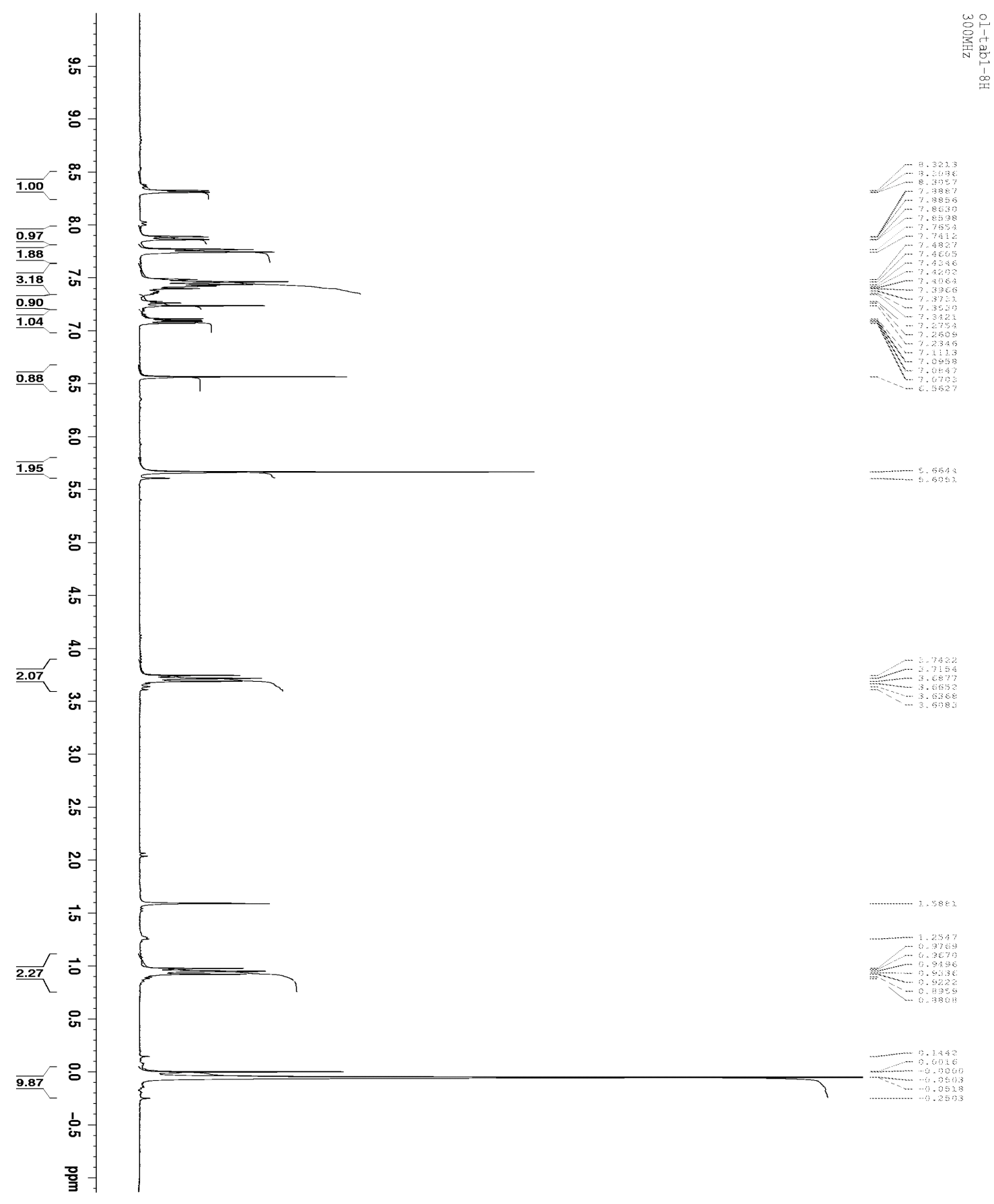



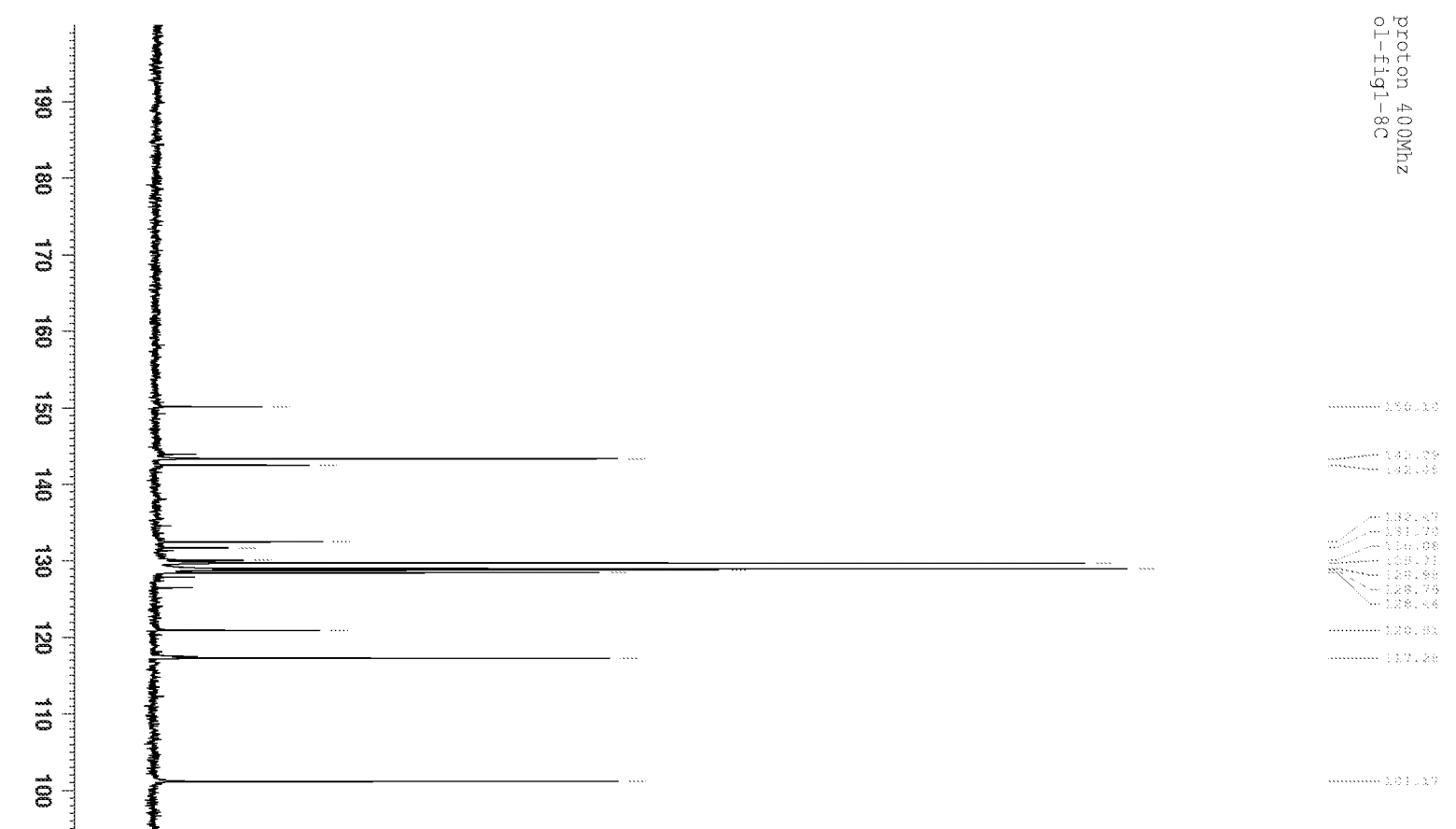

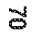

g

g

\&

$\ddot{\sigma}$

8

$\Rightarrow$

푬

重

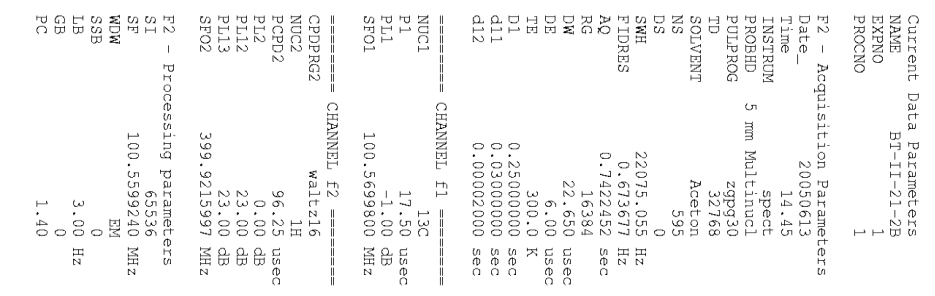


Table 2, entry 2. 2,5-Diphenyl-1-[2-(trimethylsilyl)-ethoxymethyl]-imidazole.
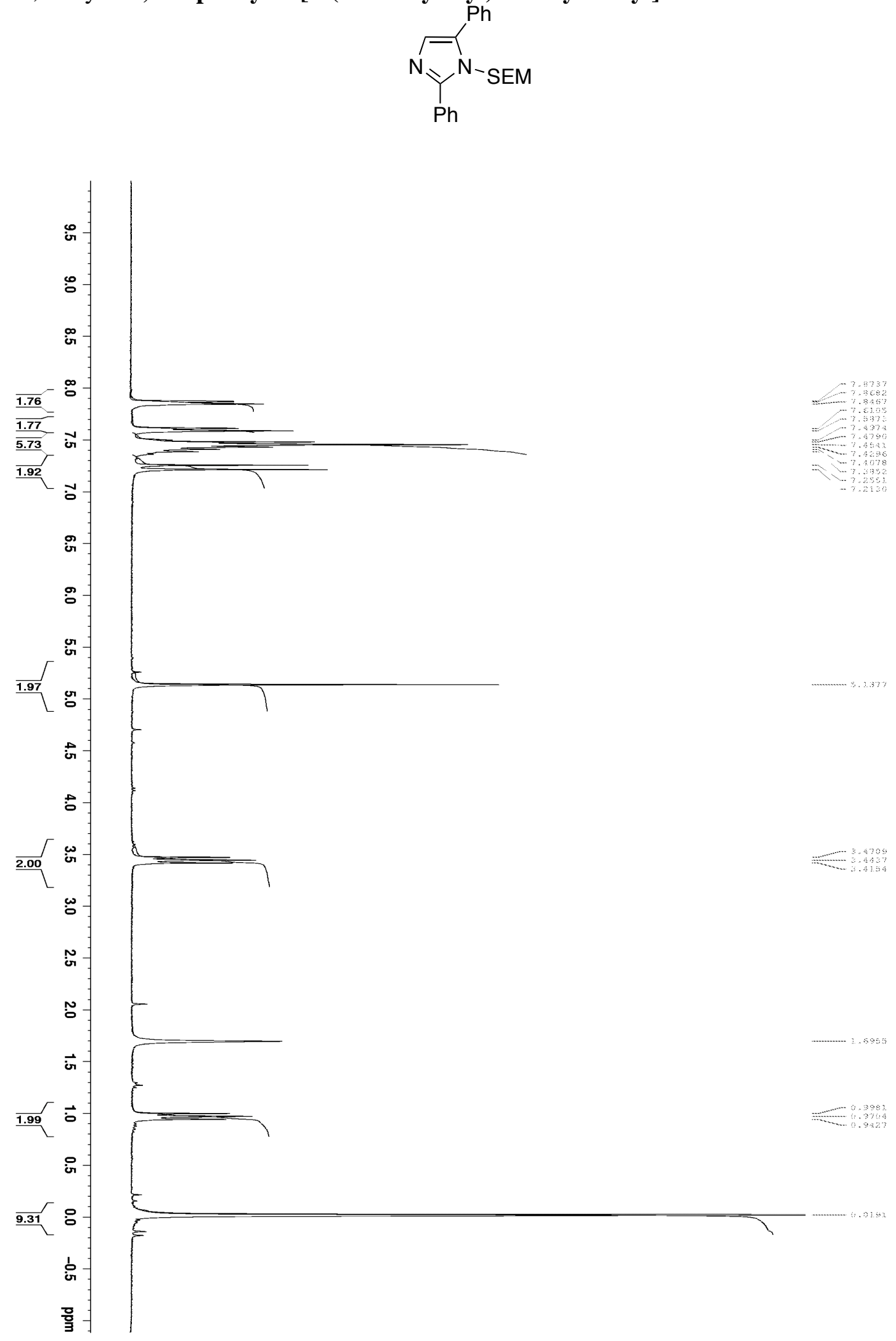
Table2 Entry 3. 5-Phenyl-1-[2-(trimethylsilyl)-ethoxymethyl]-imidazole<smiles>C#[R11]n1cncc1-c1ccccc1</smiles>

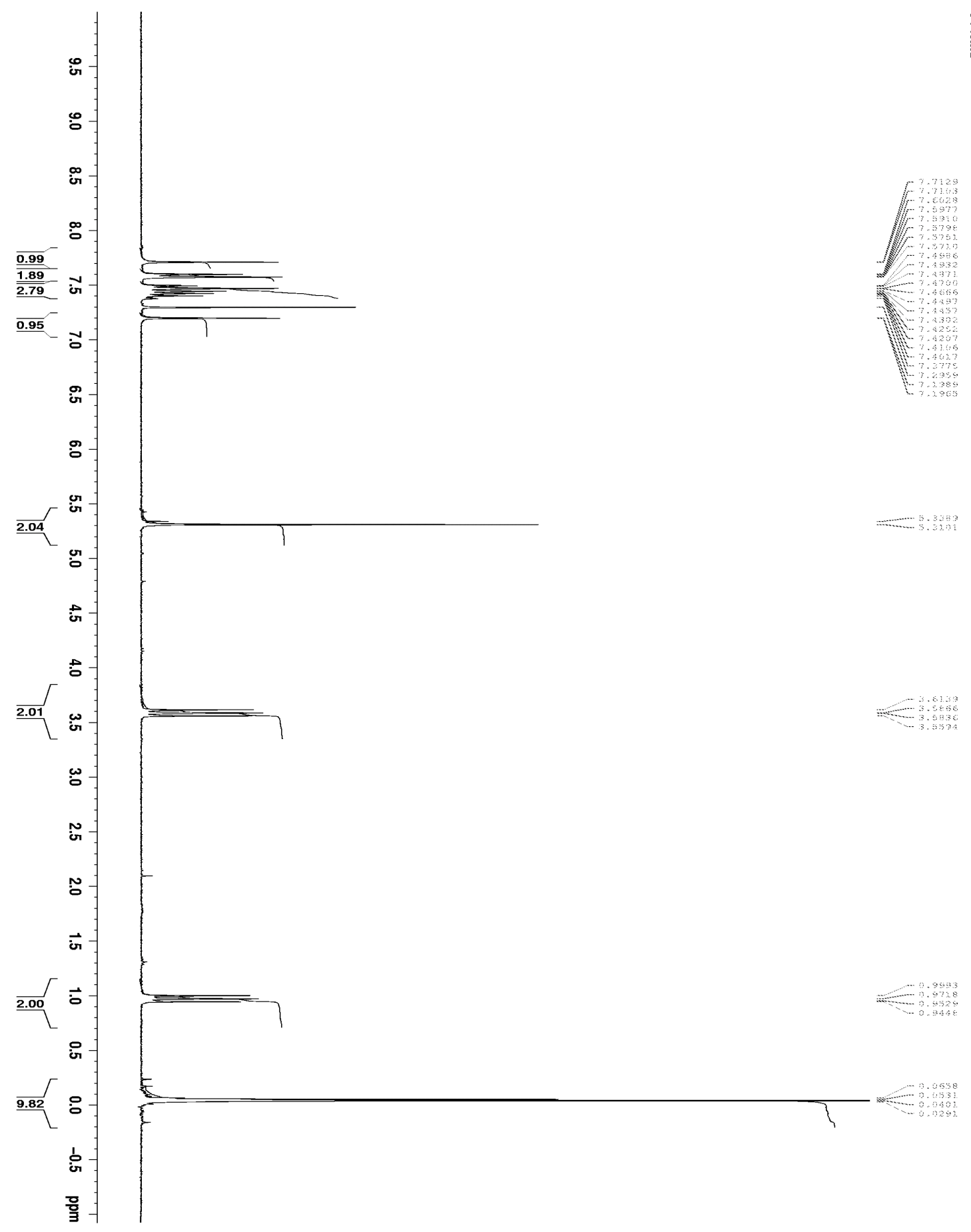



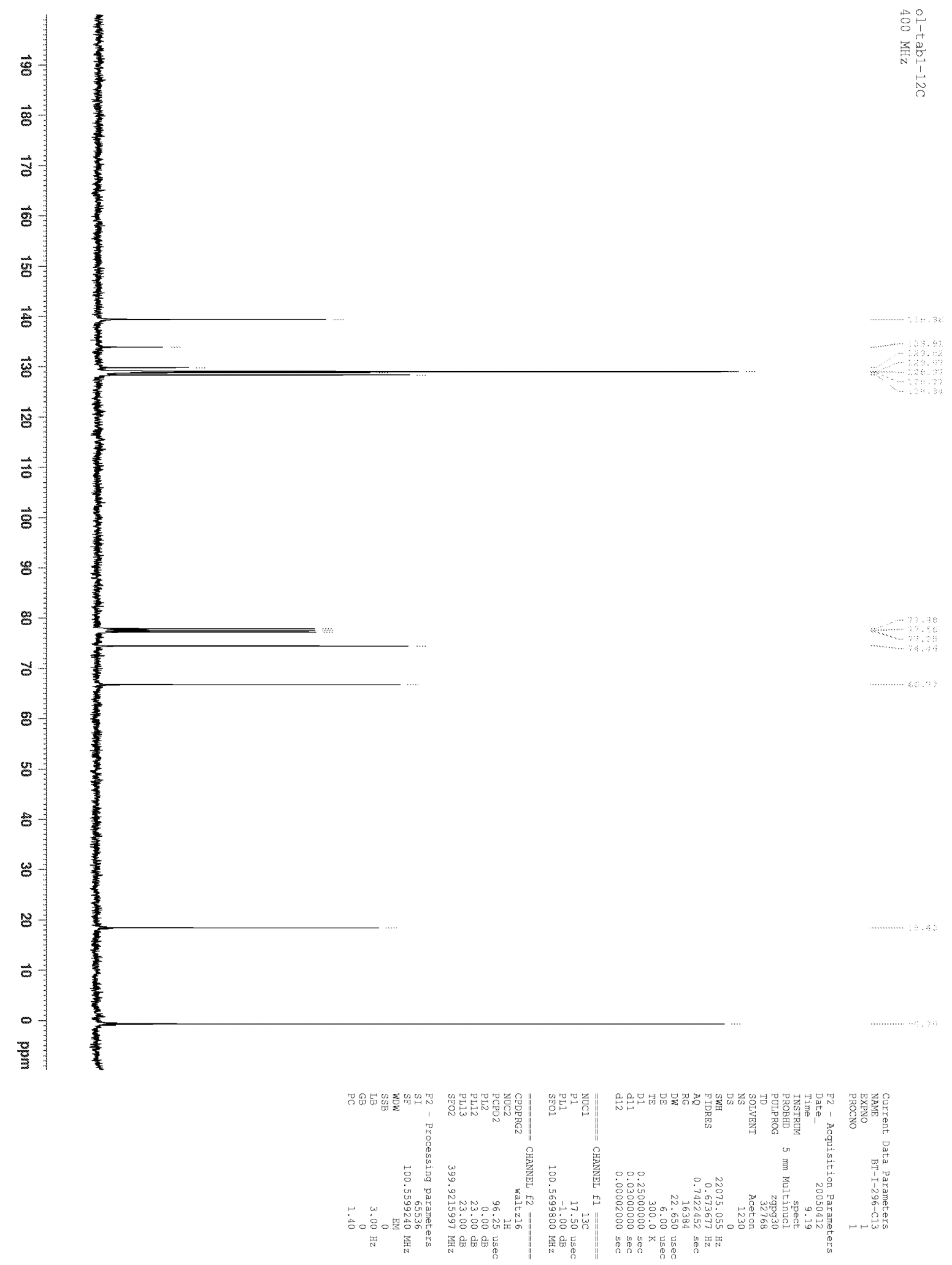
Table 3, entry 1. 3-Phenyl-imidazo[1,2-a]pyridine.
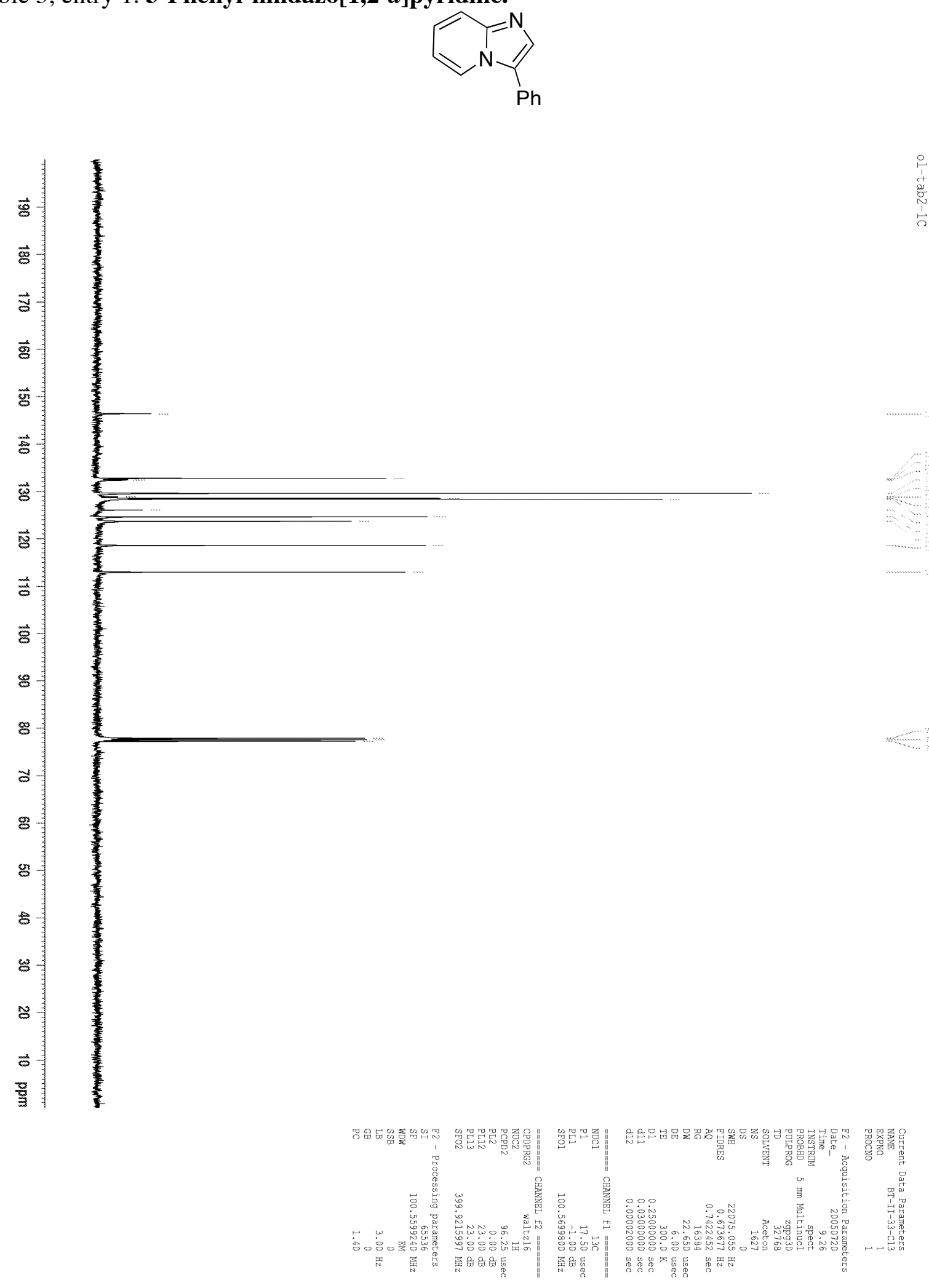
Table 3, entry 2. 3-Phenyl-imidazo[1,2-a]pyridine-2-carboxylic acid methyl ester.<smiles>CC(=O)c1nc2ccccn2c1-c1ccccc1</smiles>

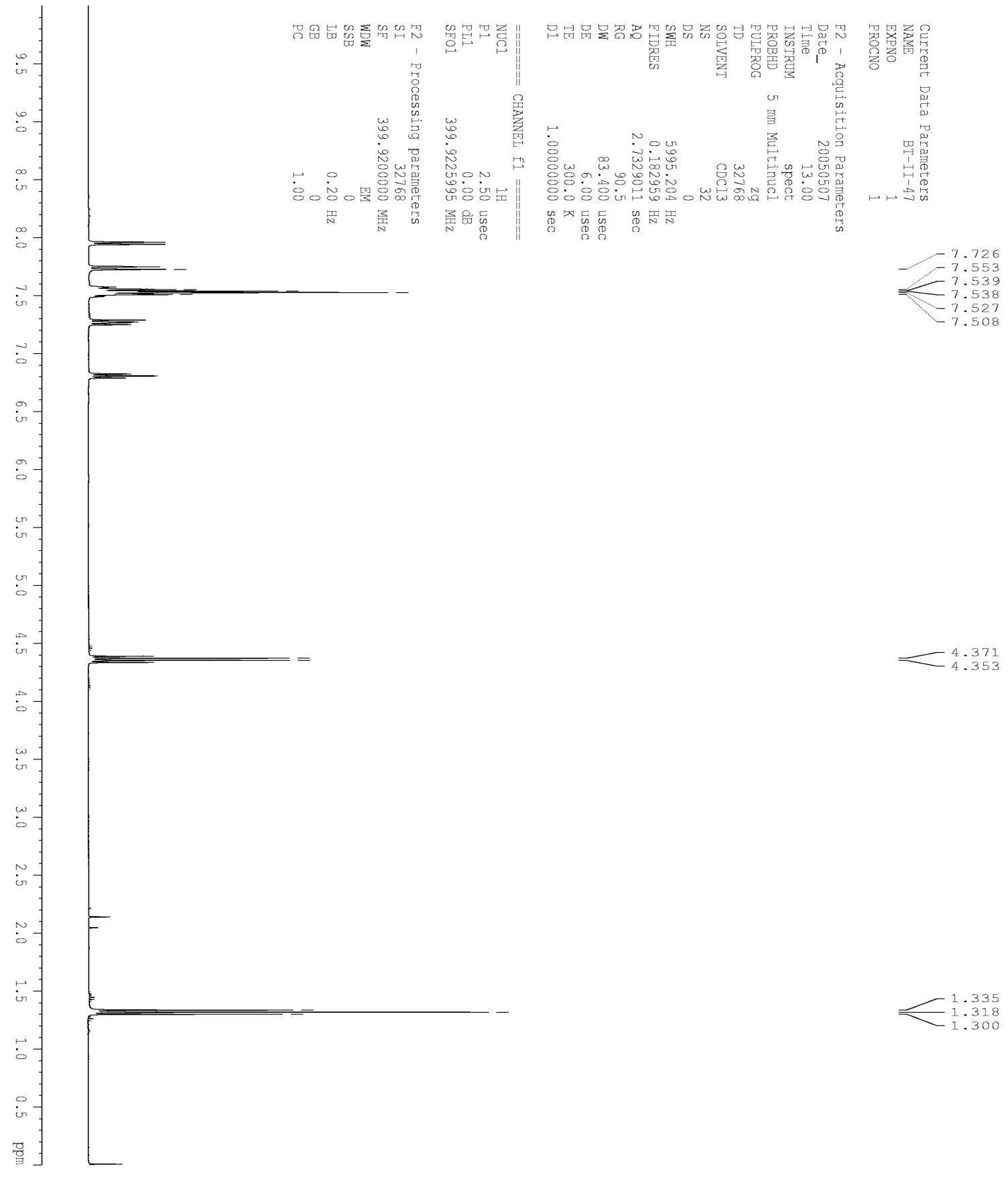


Table 3, entry 3. 3-(4-Methoxycarbonylphenyl)-imidazo[1,2-a]pyridine-2-carboxylic acid ethyl ester.
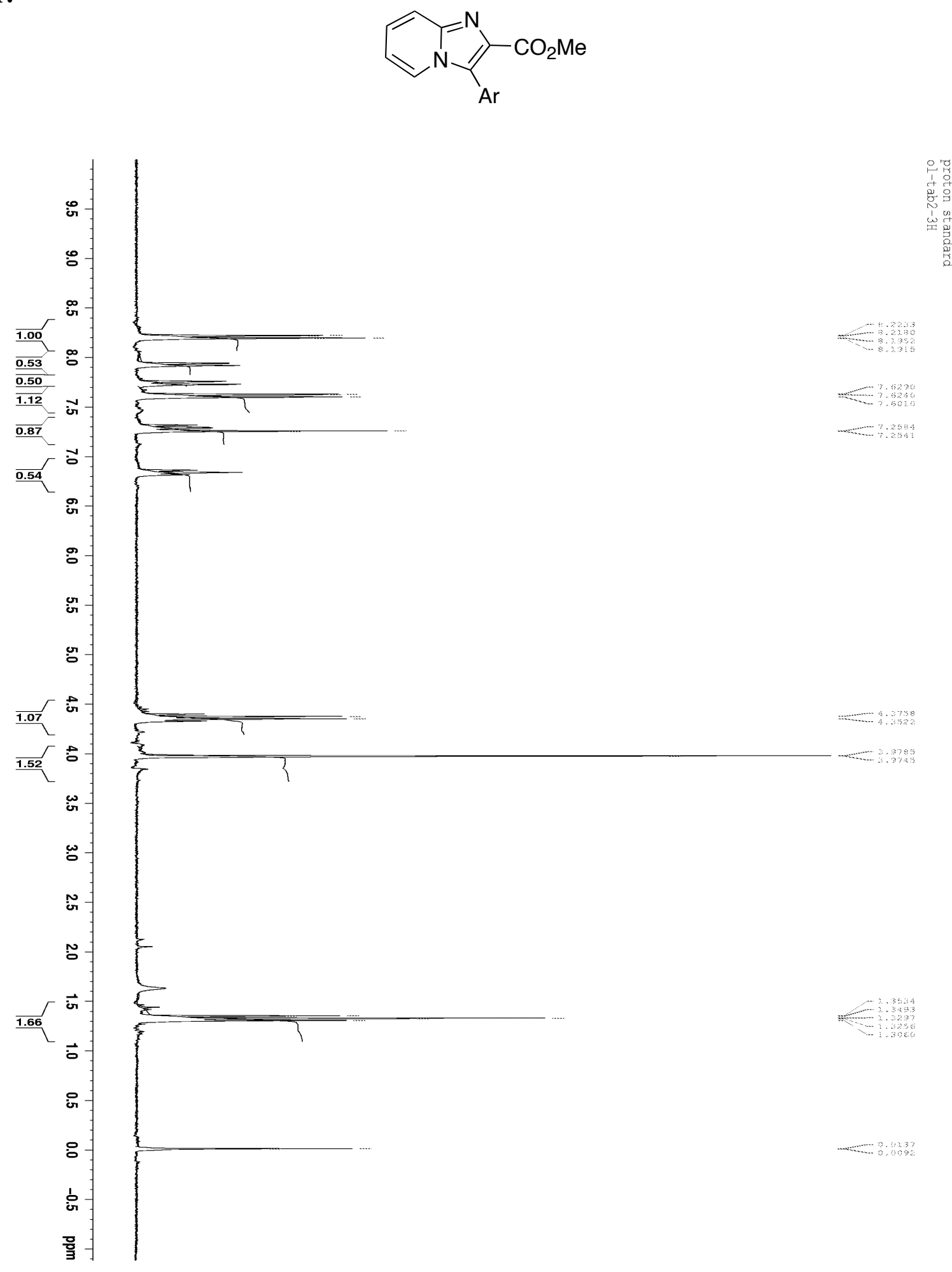


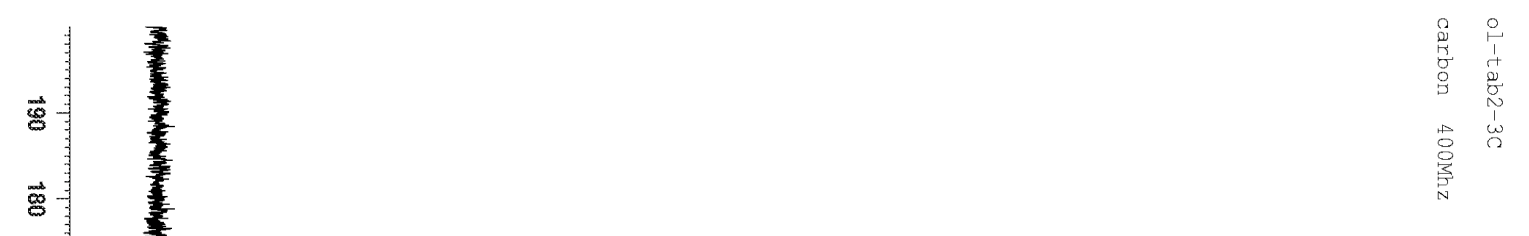

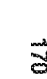

홍

풍

รี

窎

홍

$\vec{\Delta}$

8

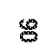

:
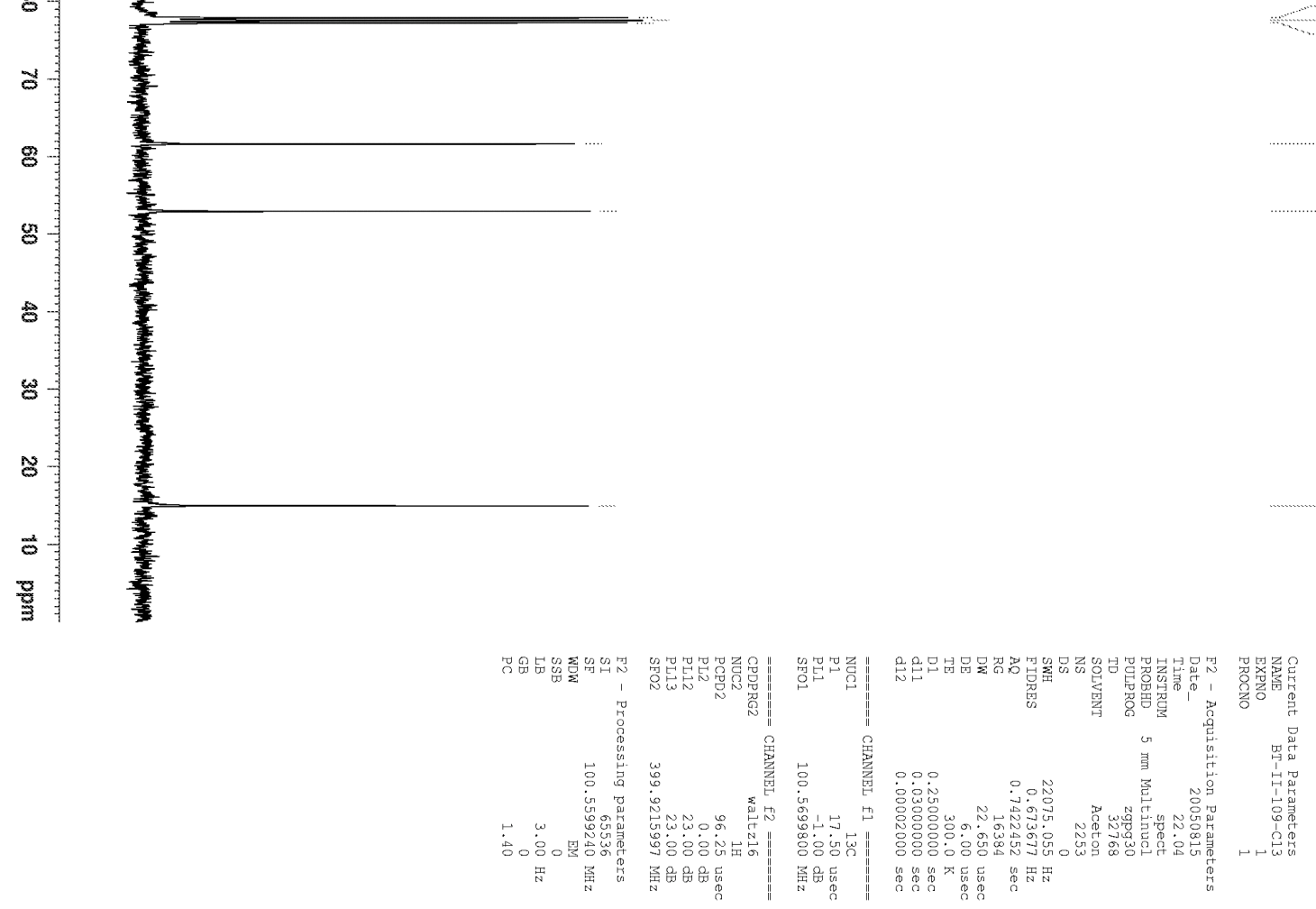